OUTER PRODUCTS AND FRAME COEFFICIENTS

\begin{tabular}{c}
\hline A Dissertation Presented to \\
the Faculty of the Graduate School \\
University of Missouri \\
\hline
\end{tabular}

\begin{tabular}{c}
\hline In Partial Fulfillment \\
of the Requirements for the Degree of \\
Doctor of Philosophy \\
\hline by \\
ERIC PINKHAM Peter G. Casazza, Dissertation Supervisor \\
MAY 2017
\end{tabular}


The undersigned, appointed by the dean of the Graduate School, have examined the dissertation entitled

\section{OUTER PRODUCTS AND FRAME COEFFICIENTS}

presented by Eric Pinkham, a candidate for the degree of Doctor of Philosophy of Mathematics, and hereby certify that in their opinion, it is worthy of acceptance:

Professor Peter G. Casazza

Professor Adam Helfer

Professor Stephen Montgomery-Smith

Professor Alina Zare 


\section{ACKNOWLEDGEMENTS}

I would like to thank all of my collaborators: Kevin Brewster, Peter Casazza, Victor Kaftal, Brian Tuomanen and Lindsey Woodland.

My committee (Peter Casazza, Adam Helfer, Stephen Mongomery-Smith and

Alina Zare) have been most patient and understanding. I greatly appreciate all of their help throughout my time at Mizzou, and I value everything I've learned from them.

My friends and family have been very good sports for putting up with all of my anxiety and supporting me through the years, and I thank them for pushing me to finish. In no particular order, I would specifically like to thank Aynsley, Daniel, Heather, Jake and Linda.

And lastly, I want to thank Pete and Janet. Pete was the best adviser that one could hope for, providing many years of fun research, interesting projects, moral support and excellent parties. 


\section{Contents}

Acknowledgements $\quad$ ii

Abstract $\quad$ v

1 Introduction 1

1.1 Notation and Conventions . . . . . . . . . . . . . . . 1

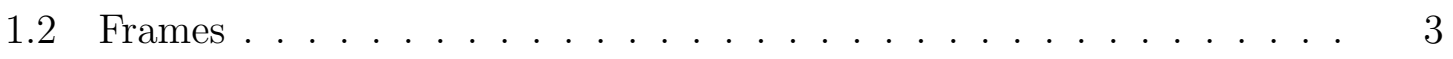

1.3 Further Properties of Frames . . . . . . . . . . . . . 6

2 Riesz Outer Product Hilbert Space Frames $\quad 8$

2.1 Some Basic Calculations . . . . . . . . . . . . . . . . . . . 9

2.2 Some Results Guaranteeing Riesz Outer Products . . . . . . . . . . . 12

2.2.1 Sparsity and Vectorized Outer Products . . . . . . . . . . 12

2.3 Computation of Riesz Bounds . . . . . . . . . . . . . . . . 14

2.4 Concrete Constructions of Riesz Bases of Outer Products . . . . . . . 18

2.5 Duals of Outer Products . . . . . . . . . . . . . . 23

2.6 Outer Cross-Products of Frames . . . . . . . . . . . . . . . . . 24

2.7 Topological Properties of Independent Outer Product Sequences . . . 27

2.8 Rank of Bordered Positive Semidefinite Matrices and Dependent Outer Product Sequences . . . . . . . . . . . . . . . . . . . 32 
2.9 The Spark of Induced Outer Product Sequences . . . . . . . . . . . . 34

3 The Distributions of Hilbert Space Frame Vectors and Frame Coef-

ficients

3.1 Estimating the Number of Non-Zero Frame Coefficients . . . . . . . . 40

3.2 The Distribution of the Frame Coefficients . . . . . . . . . . . . 43

3.3 Products of Frame Coefficients . . . . . . . . . . . . . . . . . . 48

3.4 Distance Between Vectors and Frame Vectors . . . . . . . . . . . 54

Bibliography

65

Vita

66 


\title{
OUTER PRODUCTS AND FRAME COEFFICIENTS
}

\author{
Eric Pinkham \\ Dr. Peter G. Casazza, Dissertation Supervisor
}

\begin{abstract}
In this dissertation, we will examine two distinct areas of frame theory. The first will be the area of outer products. In particular, we will examine the spanning and independence properties of the collection $\left\{\phi_{i} \phi_{i}^{*}\right\}_{i=1}^{M}$ for a given set $\left\{\phi_{i}\right\}_{i=1}^{M} \subset \mathbb{H}^{N}$. In the case that our collection of outer products is a Riesz sequence, we will examine the relation between the Riesz bounds of the outer products to those of the generating vectors. It is perhaps not surprising that an independent collection of vectors will produce an independent collection of outer products. What is surprising though, is that the outer products have the same or better Riesz bounds. However, linearly independent collections of vectors are by no means the only collections that produce independent outer products. We will see that almost all vector sequences produce independent outer product sequences, dimension and cardinality permitting.

Next we will examine the distribution of frame coefficients. We will start this investigation by examining the number of indices for which the frame coefficient is non-zero. Then we will generalize and bound these coefficients away from zero. We will study products of frame coefficients and find that in cases of particular classes of frames, we can bound a particular sum away from zero. Finally, we will look at the distance from an arbitrary vector to the frame vectors for a given frame and find some surprising results for certain classes of frames.
\end{abstract}




\section{Chapter 1}

\section{Introduction}

We start by introducing some of the basic terminology used throughout this dissertation. Though most of the necessary material is provided here, we assume that the reader has a familiarity with the basics of frame theory. The reader may wish to review $[9,11,12,13,18]$.

\subsection{Notation and Conventions}

Before we begin, we would like to standardize some notations and conventions that will be used throughout this dissertation. $\mathbb{H}^{N}$ is a real or complex Hilbert space of dimension $N$ (i.e., $\mathbb{H}^{N}=\mathbb{R}^{N}$ or $\mathbb{H}^{N}=\mathbb{C}^{N}$ ). We will generally reserve the characters $\phi, \psi, e, f, g$ for vectors, which are always column vectors. When we want to indicate the components of these vectors, we may write $\phi=(\phi(1), \ldots, \phi(N))^{T}$. We will use $1_{N} \in \mathbb{H}^{N}$ to denote the vector of all 1's (i.e. $1_{N}(i)=1$ for all $i=1, \ldots, N$ ). We will often abbreviate the set $\{1, \ldots, M\}$ as $[M]$. We will use $\phi^{*}$ to denote the conjugate transpose of $\phi$. In case of $\phi \in \mathbb{H}^{N}$ where $\mathbb{H}^{N}$ is real, we may still use $\phi^{*}$, understood that this is simply the transpose. Furthermore, we will use $\bar{\phi}$ to indicate 
the component-wise complex conjugate. Thus, for complex $\mathbb{H}^{N}$,

$$
\phi^{*}=\left[\begin{array}{c}
\overline{\phi(1)} \\
\overline{\phi(2)} \\
\vdots \\
\overline{\phi(N)}
\end{array}\right]^{T}=(\bar{\phi})^{T},
$$

and for real $\mathbb{H}^{N}$, we have $\phi^{*}=\phi^{T}$. Unless otherwise stated, $\mathbb{H}^{N}$ will be equipped with the standard real or complex inner product.

We will also be considering vector spaces of matrices, which we will write as $\mathbb{H}^{N \times N}$. Again, with a slight abuse of notation, we will write the spaces of symmetric and selfadjoint matrices as $\operatorname{sym}\left(\mathbb{H}^{N \times N}\right)$, with the understanding that we are referring to symmetric matrices over $\mathbb{R}^{N \times N}$ and self-adjoint matrices over $\mathbb{C}^{N \times N}$. That is,

$$
\operatorname{sym}\left(\mathbb{R}^{N \times N}\right)=\left\{T \in \mathbb{R}^{N \times N}: T^{T}=T\right\}
$$

and

$$
\operatorname{sym}\left(\mathbb{C}^{N \times N}\right)=\left\{T \in \mathbb{C}^{N \times N}: T^{*}=T\right\} .
$$

It is worth mentioning here that $\operatorname{sym}\left(\mathbb{R}^{N \times N}\right)$ is a real vector space of dimension $N(N+1) / 2$. However, $\operatorname{sym}\left(\mathbb{C}^{N \times N}\right)$ is not a complex vector space. Indeed, if $T \in$ $\operatorname{sym}\left(\mathbb{C}^{N \times N}\right)$, then $\sqrt{-1} T$ is not, since it is no longer self-adjoint. As such, it is a real vector space of dimension $N^{2}$. To denote the $(i, j)$ entry of a matrix $T$, we will write $T[i, j]$. Again, it may be necessary to use the component-wise complex conjugate of a matrix. That is, $\bar{T}$ is the matrix whose $(i, j)$ entry is $\overline{T[i, j]}$.

When considering vector spaces of matrices, choice of inner product is less clear. Unless otherwise stated, we will use the Frobenius inner product (often called the Hilbert-Schmidt inner product). In some sense, it is the inner product on $\mathbb{H}^{N \times N}$ that is most compatible with the standard inner product on $\mathbb{H}^{N}$. 
Definition 1.1.1. Let $S, T \in \mathbb{H}^{N \times N}$. The Frobenius inner product is

$$
\langle S, T\rangle_{F}=\operatorname{tr}\left(S^{*} T\right)=\operatorname{tr}\left(S T^{*}\right)=\sum_{i=1}^{N} \sum_{j=1}^{N} S[i, j] \overline{T[i, j]} .
$$

We may drop the subscript $F$ when there is no danger of confusion.

\subsection{Frames}

Definition 1.2.1. A sequence of vectors $\left\{\phi_{i}\right\}_{i=1}^{M} \subset \mathbb{H}^{N}$ is a frame for $\mathbb{H}^{N}$ provided that there exists $0<A \leq B<\infty$ such that

$$
A\|\psi\|^{2} \leq \sum_{i=1}^{M}\left|\left\langle\phi_{i}, \psi\right\rangle\right|^{2} \leq B\|\psi\|^{2}
$$

for all $\psi \in \mathbb{H}^{N}$. A and $B$ are called lower and upper frame bounds, respectively.

In the finite dimensional setting, a collection of vectors is a frame if and only if it is a spanning set (see [13]). When $M>N$, we say the frame is redundant. It should be noted that there is a continuum of frame bounds for a redundant frame. The largest lower frame bound and the smallest upper frame bound are the optimal frame bounds. We characterize several classes of frames of particular interest.

Definition 1.2.2. Let $\left\{\phi_{i}\right\}_{i=1}^{M}$ be a frame for $\mathbb{H}^{N}$ with frame bounds $A$ and $B$.

(i) If $A=B$ is possible, then $\left\{\phi_{i}\right\}_{i=1}^{M}$ is an A-tight frame (or simply a tight frame). If $A=B=1$ is possible, then $\left\{\phi_{i}\right\}_{i=1}^{M}$ is a Parseval frame.

(ii) If $\left\|\phi_{i}\right\|=1$ for all $i \in[M]$, then $\left\{\phi_{i}\right\}_{i=1}^{M}$ is a unit norm frame.

(iii) $\left\{\left\langle x, \phi_{i}\right\rangle\right\}_{i=1}^{M}$ are called the frame coefficients of the vector $x \in \mathcal{H}_{N}$ with respect to frame $\left\{\phi_{i}\right\}_{i=1}^{M}$. 
(iv) If the frame is unit norm and there is a constant $d$ so that $\left|\left\langle\phi_{i}, \phi_{j}\right\rangle\right|=d$ for all $1 \leq i \neq j \leq M$, then $\left\{\phi_{i}\right\}_{i=1}^{M}$ is an equiangular frame.

There are several operators of particular importance to the study of frames.

Definition 1.2.3. Let $\Phi=\left\{\phi_{i}\right\}_{i=1}^{M}$ be a frame for $\mathbb{H}^{N}$.

(i) The synthesis operator of $\Phi$ is

$$
T: \ell_{2}^{M} \rightarrow \mathbb{H}^{N} \quad T:\left(a_{i}\right)_{i=1}^{M} \mapsto \sum_{i=1}^{M} a_{i} \phi_{i} .
$$

Its matrix representation is

$$
T=\left[\begin{array}{cccc}
\mid & \mid & & \mid \\
\phi_{1} & \phi_{2} & \cdots & \phi_{M} \\
\mid & \mid & & \mid
\end{array}\right] .
$$

(ii) The analysis operator of $\Phi$ is the Hermitian adjoint of $T$,

$$
T^{*}: \mathbb{H}^{N} \rightarrow \ell_{2}^{M} \quad T^{*}: \psi \mapsto\left(\left\langle\psi, \phi_{i}\right\rangle\right)_{i=1}^{N}
$$

(iii) The frame operator of $\Phi$ is $S=T T^{*}$ so that

$$
S: \mathbb{H}^{N} \rightarrow \mathbb{H}^{M} \quad S: \psi \mapsto \sum_{i=1}^{M}\left\langle\psi, \phi_{i}\right\rangle \phi_{i}
$$

(iv) The Gram matrix of $\Phi$ is

$$
G(\Phi)=T^{*} T=\left[\left\langle\phi_{j}, \phi_{i}\right\rangle\right]_{i, j=1}^{M} .
$$

It follows that the non-zero eigenvalues of $S$ and $G(\Phi)$ are equal, so the largest and smallest non-zero eigenvalues of $G(\Phi)$ are the optimal upper and lower frame bounds of $\Phi$, respectively.

The frame operator is useful for understanding the frame from which it arose. 
Theorem 1.2.4. Let $\left\{\phi_{i}\right\}_{i=1}^{M}$ be a frame for $\mathbb{H}^{N}$. Then the frame operator $S$ is selfadjoint, positive, and invertible. Furthermore, the largest and smallest eigenvalues of $S$ are precisely the optimal upper and lower frame bounds of $\left\{\phi_{i}\right\}_{i=1}^{M}$, respectively.

Reconstruction is carried out by

$$
\psi=S S^{-1} \psi=\sum_{i=1}^{M}\left\langle\psi, \phi_{i}\right\rangle S^{-1} \phi_{i}=\sum_{i=1}^{M}\left\langle\psi, S^{-1} \phi_{i}\right\rangle \phi_{i} .
$$

This is a useful representation of any vector in our Hilbert space through the frame operator. For applications, we want the frame operator to be as well conditioned as possible for stability of the representation. This means that frames that are close to being tight are more desirable than those with large a condition number (i.e., large $B / A$ ). Tight frames are particularly useful for encoding and decoding as above. The frame operator of a tight frame is a scalar multiple of the identity, and so inverting them is trivial. This is especially useful when our space has very high dimension, as is common in applications.

The vectors $\left\{S^{-1} \phi_{i}\right\}_{i=1}^{M}$ themselves form a frame for $\mathbb{H}^{N}$. We summarize its properties in the following proposition (see [12]):

Proposition 1.2.5. Let $\left\{\phi_{i}\right\}_{i=1}^{M}$ be a frame for $\mathbb{H}^{N}$ with frame operator $S$ and frame bounds $A$ and $B$. Then $\left\{S^{-1} \phi_{i}\right\}_{i=1}^{M}$ is a frame for $\mathbb{H}^{N}$ with frame operator $S^{-1}$ and frame bounds $B^{-1}$ and $A^{-1}$.

The frame $\left\{S^{-1} \phi_{i}\right\}_{i=1}^{M}$ is said to be dual to $\left\{\phi_{i}\right\}_{i=1}^{M}$ and is, in fact, called the cannonical dual frame to $\left\{\phi_{i}\right\}_{i=1}^{M}$.

Definition 1.2.6. Let $\Phi=\left\{\phi_{i}\right\}_{i=1}^{M}$ be a frame for $\mathbb{H}^{N}$. $\left\{\psi_{i}\right\}_{i=1}^{M}$ is a dual frame for 
$\Phi$ if

$$
x=\sum_{i=1}^{M}\left\langle x, \psi_{i}\right\rangle \phi_{i}=\sum_{i=1}^{M}\left\langle x, \phi_{i}\right\rangle \psi_{i}
$$

for all $x \in \mathbb{H}^{N}$.

In general, there are many dual frames for any given frame, provided $M>N$. In fact, for a frame $\left\{\phi_{i}\right\}_{i=1}^{M}$ with synthesis operator $T$, the dual frames are determined precisely by the left inverses of $T^{*}$ (see [12]).

\subsection{Further Properties of Frames}

We will briefly discuss some important properties of certain types of frames introduced in the previous section.

Proposition 1.3.1. If $\left\{\phi_{i}\right\}_{i=1}^{M}$ is an equal norm Parseval frame for $\mathbb{H}^{N}$, then $\left\|\phi_{i}\right\|^{2}=$ $N / M$ for all $i$.

Proof. Let $\left\{\phi_{i}\right\}_{i=1}^{M}$ be an equal norm Parseval frame for $\mathbb{H}^{N}$. Then we know its frame operator $S$ is the identity. Thus,

$$
N=\operatorname{tr}(I)=\operatorname{tr}\left(T T^{*}\right)=\operatorname{tr}\left(T^{*} T\right) .
$$

From which it follows that $\sum_{i=1}^{N}\left\|\phi_{i}\right\|^{2}=N$ and $\left\|\phi_{i}\right\|^{2}=N / M$.

From the above, we get the following property of unit norm tight frames:

Proposition 1.3.2. Suppose that $\left\{\phi_{i}\right\}_{i=1}^{M}$ is a unit norm tight frame for $\mathbb{H}^{N}$, then its frame bound is $M / N$.

Proposition 1.3.3. If $\left\{\phi_{i}\right\}_{i=1}^{M}$ is a Parseval frame for $\mathbb{H}^{N}$, then $\left\{\phi_{i}\right\}_{i=1}^{M}$ is self-dual. 
While Parseval frames enjoy some remarkable properties, they are, in fact, fairly easy to construct.

Proposition 1.3.4. Let $\left\{\phi_{i}\right\}_{i=1}^{M}$ be a frame for $\mathbb{H}^{N}$ with frame operator $S$. Then $\left\{S^{-1 / 2} \phi_{i}\right\}_{i=1}^{M}$ is a Parseval frame for $\mathbb{H}^{N}$.

Equiangular tight frames are one of the most restrictive classes of frames. Their construction- even proof of their existence or non-existence- is difficult. For example, there is only one frame (up to an equivalence class) for $\mathbb{R}^{N}$ with $N+1$ elements and there are no equiangular tight frames with 5 elements in $\mathbb{R}^{3}$ (see for example [18]). Perhaps one of the reasons this is so is that their vectors achieve the so called Welch bound. The Welch bound was introduced in [19] as a lower bound on the maximum cross correlation between signals. For frames, this means the following:

Theorem 1.3.5. Let $\left\{\phi_{i}\right\}_{i=1}^{M}$ be an equiangular tight frame for $\mathbb{H}^{N}$. Then

$$
\left|\left\langle\phi_{i}, \phi_{j}\right\rangle\right|^{2}=\frac{M-N}{N(M-1)} .
$$

There is one final property for frames that we will introduce here.

Definition 1.3.6. Let $\left\{\phi_{i}\right\}_{i=1}^{M}$ be a frame for $\mathbb{H}^{N}$. We define the spark of $\left\{\phi_{i}\right\}_{i=1}^{M}$, written $\operatorname{Spark}\left(\left\{\phi_{i}\right\}_{i=1}^{M}\right)$, as the size of the smallest linearly dependent subset of $\left\{\phi_{i}\right\}_{i=1}^{M}$. $\left\{\phi_{i}\right\}_{i=1}^{M}$ is said to be full spark if $\operatorname{Spark}\left(\left\{\phi_{i}\right\}_{i=1}^{M}\right)=N+1$.

Intuitively, a frame for $\mathbb{H}^{N}$ is full spark if every subset of size $N$ is linearly independent. 


\section{Chapter 2}

\section{Riesz Outer Product Hilbert Space Frames}

The following was a joint project with Peter Casazza and Brian Tuomanen (see [10] for more on this topic). In this chapter we will examine the properties of the outer products induced by a frame. Outer products can be abstractly considered as tensors, but in our case we will think of them as rank-one projections. Outer products have recently appeared in numerous papers (see, $[6,16])$ addressing the scaling problem. In this chapter we will focus on the notion of Riesz outer product sequences. We start with some definitions.

Definition 2.0.1. A sequence of vectors $\left\{\phi_{i}\right\}_{i=1}^{M} \subset \mathbb{H}^{N}$ is a Riesz sequence provided there exists $0<A \leq B<\infty$ such that

$$
A \sum_{i=1}^{M}\left|a_{i}\right|^{2} \leq\left\|\sum_{i=1}^{M} a_{i} \phi_{i}\right\|^{2} \leq B \sum_{i=1}^{M}\left|a_{i}\right|^{2}
$$

for all $\left(a_{i}\right)_{i=1}^{M} \in \mathbb{H}^{M}$. A and $B$ are called lower and upper Riesz bounds, respectively.

When dealing with finite dimensional vector spaces, these objects have a very simple characterization: a set is Riesz if and only if it is linearly independent. We will use independent and Riesz nearly interchangeably in this chapter. We will use Riesz when we are particularly concerned about the Riesz bounds. 
The second object we need to define is the outer product of two vectors.

Definition 2.0.2. For $\phi, \psi \in \mathbb{H}^{N}$, define the outer product of $\phi$ and $\psi$ by $\phi \psi^{*}$ in terms of standard matrix multiplication. For any vector $\phi \in \mathbb{H}^{N}$, we define the induced outer product of $\phi$ as $\phi \phi^{*}$. Note that if $\phi$ is a unit norm vector, then $\phi \phi^{*}$ will be a rank-one orthogonal projection.

Again, we need to be careful when we consider the "space of outer products." The set of outer products is not a vector space but rather a subset of $\operatorname{sym}\left(\mathbb{H}^{N \times N}\right)$. Indeed, the sum of two rank-one projections need not be rank-one. We will see, however, that there are bases of outer products for $\operatorname{sym}\left(\mathbb{H}^{N \times N}\right)$.

\subsection{Some Basic Calculations}

We start with a simple calculation.

Lemma 2.1.1. For any vectors $\phi_{1}, \phi_{2} \in \mathbb{H}^{N}$ we have

$$
\left\langle\phi_{1} \phi_{1}^{*}, \phi_{2} \phi_{2}^{*}\right\rangle_{F}=\left|\left\langle\phi_{1}, \phi_{2}\right\rangle\right|^{2}
$$

Proof. We compute:

$$
\begin{aligned}
\left\langle\phi_{1} \phi_{1}^{*}, \phi_{2} \phi_{2}^{*}\right\rangle_{F} & =\operatorname{Tr}\left(\phi_{2} \phi_{2}^{*} \phi_{1} \phi_{1}^{*}\right) \\
& =\operatorname{Tr}\left(\phi_{2}\left\langle\phi_{1}, \phi_{2}\right\rangle \phi_{1}^{*}\right) \\
& =\operatorname{Tr}\left(\left\langle\phi_{1}, \phi_{2}\right\rangle\left\langle\phi_{2}, \phi_{1}\right\rangle\right) \\
& =\left|\left\langle\phi_{1}, \phi_{2}\right\rangle\right|^{2} .
\end{aligned}
$$

Corollary 2.1.2. $\phi_{1} \perp \phi_{2}$ in $\mathbb{H}^{N}$ if and only if $\phi_{1} \phi_{1}^{*} \perp \phi_{2} \phi_{2}^{*}$ in $\operatorname{sym}\left(\mathbb{H}^{N \times N}\right)$. 
The Gram matrices of our vectors will be one of the main tools in examining the outer products of a collection of vectors. When dealing with a Riesz sequence or a linearly independent collection of vectors, the Gram matrix will be positive-definite. Furthermore, the largest and smallest eigenvalues of this matrix represent the optimal upper and lower Riesz bounds of our sequence, respectively. In the case of redundant frames, the Gram matrix is singular. However, the largest and smallest non zero eigenvalues give the upper and lower frame bounds, respectively. We will need to define the the Gram matrix of outer products.

Theorem 2.1.3. Let $\left\{\phi_{i}\right\}_{i=1}^{M}$ be a sequence of vectors in $\mathbb{H}^{N}$. Then the Gram matrix of $\left\{\phi_{i} \phi_{i}^{*}\right\}_{i=1}^{M}$ is

$$
G=\left[\left|\left\langle\phi_{i}, \phi_{j}\right\rangle\right|^{2}\right] .
$$

Moreover,

1. If $\left\{\phi_{i} \phi_{i}^{*}\right\}_{i=1}^{M}$ is a Riesz sequence, then the optimal Riesz bounds are the largest and smallest eigenvalues of $G$.

2. If $\left\{\phi_{i} \phi_{i}^{*}\right\}_{i=1}^{M}$ is a frame, then the frame bounds are the largest and smallest nonzero eigenvalues of $G$.

The Gram matrix of the induced outer products can be represented in terms of the Gram matrix of the original vectors by using the Hadamard product.

Definition 2.1.4. Given two matrices $A$ and $B$ in $\mathbb{H}^{M \times N}$ with entries $A[i, j]$ and $B[i, j]$, respectively, the Hadamard product of $A$ and $B$ is

$A \circ B=C$ 
where $C[i, j]=A[i, j] B[i, j]$.

The following is a well-known theorem about Hadamard products (see [15] for example):

Theorem 2.1.5. Let $A$ and $B$ be positive semidefinite. Any eigenvalue $\lambda(A \circ B)$ of $A \circ B$ satisfies

$$
\begin{aligned}
\lambda_{\min }(A) \lambda_{\min }(B) & \leq\left(\min _{i} A[i, i]\right) \lambda_{\min }(B) \\
& \leq \lambda(A \circ B) \\
& \leq\left(\max _{i} A[i, i]\right) \lambda_{\max }(B) \\
& \leq \lambda_{\max }(A) \lambda_{\max }(B) .
\end{aligned}
$$

Corollary 2.1.6. If $\left\{\phi_{i}\right\}_{i=1}^{M}$ is a unit norm Riesz sequence with Riesz bounds $A$ and $B$, then $\left\{\phi_{i} \phi_{i}^{*}\right\}_{i=1}^{M}$ is also Riesz with the same Riesz bounds.

Proof. Let $G$ be the Gram matrix of $\left\{\phi_{i}\right\}_{i=1}^{M}$ and $H$ be the Gram matrix of the induced outer products. Then

$$
H=G \circ \bar{G}=G \circ G^{T} .
$$

Since $G$ and $G^{T}$ have the same eigenvalues and the diagonal entries of $G$ are $\left\|\phi_{i}\right\|^{2}$, the result follows.

It may not be surprising that unit norm Riesz sequences produce Riesz outer products. What is surprising is that the same Riesz bounds hold. That is, Riesz bounds cannot worsen when moving to the outer product space. A natural question to ask at this point is whether the Riesz bounds of the induced outer products can be better than the Riesz bounds of the original vectors. The answer is yes. 
Example 2.1.7. Let $\phi_{1}=[0,1]^{T}, \phi_{2}=[\sqrt{\varepsilon}, \sqrt{1-\varepsilon}]^{T}$ for $0<\varepsilon<1$. Then $\left\{\phi_{i}\right\}_{i=1}^{2}$ is Riesz with Riesz bounds $1-\sqrt{\varepsilon}$ and $1+\sqrt{\varepsilon}$, while $\left\{\phi_{i} \phi_{i}^{*}\right\}_{i=1}^{2}$ is Riesz with bounds $1-\varepsilon$ and $1+\varepsilon$.

Proof. The Gram matrix of $\left\{\phi_{1}, \phi_{2}\right\}$ is

$$
\left[\begin{array}{cc}
1 & \sqrt{\varepsilon} \\
\sqrt{\varepsilon} & 1
\end{array}\right]
$$

while that of $\left\{\phi_{1} \phi_{1}^{*}, \phi_{2} \phi_{2}^{*}\right\}$ is

$$
\left[\begin{array}{ll}
1 & \varepsilon \\
\varepsilon & 1
\end{array}\right]
$$

The eigenvalues of these matrices are as required.

\subsection{Some Results Guaranteeing Riesz Outer Prod- ucts}

The preceding sections show the difficulty in deciding whether a dependent collection of vectors produces independent outer products. Later, we will see that in some sense most families of vectors induce independent outer product sequences. But it is more important to determine which frames induce dependent outer products. We will see a full characterization of all frames that induce dependent outer products. For now, we give a few simple observations that can be used to quickly check whether a sequence will produce independent outer products.

\subsubsection{Sparsity and Vectorized Outer Products}

Definition 2.2.1. Let $\phi \in \mathbb{H}^{N}$. Define the vectorization of $\phi \phi^{*}$ as the vector obtained by stacking the columns of $\phi \phi^{*}$ on top of each other. That is, the vectorization of $\phi \phi^{*}$ 
is

$$
\left[\begin{array}{c}
\phi(1) \bar{\phi} \\
\phi(2) \bar{\phi} \\
\vdots \\
\phi(N) \bar{\phi}
\end{array}\right]
$$

where $\phi(k)$ is the kth entry of $\phi$.

Proposition 2.2.2. Let $\left\{\phi_{i}\right\}_{i=1}^{M}$ be a frame for $\mathbb{H}^{N}$ with no zero vectors. For $k=$ $1, \ldots, N$, define $I_{k}=\left\{i: \phi_{i}(k) \neq 0\right\}$. If $\left\{\phi_{i}\right\}_{i \in I_{k}}$ is independent for all $k$, then $\left\{\phi_{i} \phi_{i}^{*}\right\}_{i=1}^{M}$ is independent.

Proof. Let $\left\{\phi_{i}\right\}_{i=1}^{M}$ be a frame with the properties as stated. Let $C_{i}$ be the vectorization of $\phi_{i} \phi_{i}^{*}$. Now consider the synthesis operator of $\left\{C_{i}\right\}_{i=1}^{M}$ :

$$
\left[\begin{array}{ccccc}
\phi_{1}(1) \overline{\phi_{1}} & \phi_{2}(1) \overline{\phi_{2}} & \phi_{3}(1) \overline{\phi_{3}} & \cdots & \phi_{M}(1) \overline{\phi_{M}} \\
\phi_{1}(2) \overline{\phi_{1}} & \phi_{2}(2) \overline{\phi_{2}} & \phi_{3}(2) \overline{\phi_{3}} & \cdots & \phi_{M}(2) \overline{\phi_{M}} \\
\vdots & \vdots & \vdots & & \vdots \\
\phi_{1}(N) \overline{\phi_{1}} & \phi_{2}(N) \overline{\phi_{2}} & \phi_{3}(N) \overline{\phi_{3}} & \cdots & \phi_{M}(N) \overline{\phi_{M}}
\end{array}\right] .
$$

Notice that since $0 \notin\left\{\phi_{i}\right\}_{i=1}^{M}$, each $\phi_{i}$ contains at least one non-zero entry, say $\phi_{i}(k) \neq 0$. Then, since $\phi_{i}(k) \overline{\phi_{i}}$ is part of $C_{i}$, we have that $C_{i} \neq 0$ for all $i$.

Now suppose that there exists scalars $a_{i}$ (not all zero) such that

$$
\sum_{i=1}^{M} a_{i} C_{i}=0
$$

Then there is at least one $l$ such that $a_{l} C_{l} \neq 0$. Then, by hypothesis, there is a row $k$ such that $\sum_{i} a_{i} \phi_{i}(k) \overline{\phi_{i}}=0$ but $a_{l} \phi_{l}(k) \overline{\phi_{l}} \neq 0$. Then

$$
\sum_{i \in I_{k}} a_{i} \phi_{i}(k) \overline{\phi_{i}}=0
$$

which contradicts our assumption that $\left\{\phi_{i}\right\}_{i \in I_{k}}$ is linearly independent. 
Remark 2.2.3. The conditions of the above proposition are fairly constrictive, but, in certain cases, the above proposition can be useful. It will be used later to quickly verify examples.

Corollary 2.2.4. Let $\left\{\phi_{i}\right\}_{i=1}^{M}$ be a frame for which every subset of size $k$ is linearly independent. If the rows of the analysis operator are $k$-sparse (i.e. have at most $k$ non-zero entries) then the induced outer products are linearly independent.

\subsection{Computation of Riesz Bounds}

In the following section, we will examine more closely the Riesz bounds of the induced outer products. Here we give the "optimal" Riesz bounds for a set of unit norm vectors and sufficient conditions to achieve them.

The following is immediate by Lemma 2.1.1.

Proposition 2.3.1. Let $\left\{\phi_{i}\right\}_{i=1}^{M}$ be vectors in $\mathbb{H}^{N}$. The sequence $\left\{\phi_{i} \phi_{i}^{*}\right\}_{i=1}^{M}$ is orthonormal if and only if $\left\{\phi_{i}\right\}_{i=1}^{M}$ is orthonormal.

Since a redundant frame cannot produce a Riesz sequence with tight Riesz bounds, one might ask how close we can get. Before computing the optimal Riesz bounds of a set of rank-one projections, we need to introduce the frame potential.

Definition 2.3.2. Let $\left\{\phi_{i}\right\}_{i=1}^{M}$ be a frame in $\mathbb{H}^{N}$. The frame potential is

$$
\operatorname{FP}\left(\left\{\phi_{i}\right\}_{i=1}^{M}\right)=\sum_{i=1}^{M} \sum_{j=1}^{M}\left|\left\langle\phi_{i}, \phi_{j}\right\rangle\right|^{2} .
$$

Proposition 2.3.3. The frame potential of a unit norm tight frame with $M$ elements in $\mathbb{H}^{N}$ is $M^{2} / N$, which is a minimum over all unit norm frames.

See $[7,13]$ for a proof of the above result. 
Theorem 2.3.4. If $\left\{\phi_{i}\right\}_{i=1}^{M}$ is a unit norm frame for $\mathbb{H}^{N}$, then the upper Riesz bound of $\left\{\phi_{i} \phi_{i}^{*}\right\}_{i=1}^{M}$ is at least $M / N$. Moreover, we have equality if and only if $\left\{\phi_{i}\right\}_{i=1}^{M}$ is a unit norm tight frame.

Proof. If $\left\{\phi_{i}\right\}_{i=1}^{M}$ is a unit norm frame whose outer products have Gram matrix $G$, then

$$
\begin{aligned}
\frac{M}{N} & \leq \frac{1}{M} \operatorname{FP}\left(\left\{\phi_{i}\right\}_{i=1}^{M}\right) \\
& =\frac{1}{M}\left\|\left(\sum_{i=1}^{M}\left|\left\langle\phi_{i}, \phi_{j}\right\rangle\right|^{2}\right)_{j=1}^{M}\right\|_{\ell_{1}}\left\|\left(\sum_{i=1}^{M}\left|\left\langle\phi_{i}, \phi_{j}\right\rangle\right|^{2}\right)_{j=1}^{M}\right\|_{\ell_{2}} \\
& \leq \frac{1}{M} \sqrt{M}\|\|_{j=1}^{M} \|_{\ell_{2}} \\
& =\left\|\left(\frac{1}{\sqrt{M}} \sum_{i=1}^{M}\left|\left\langle\phi_{i}, \phi_{j}\right\rangle\right|^{2}\right)^{T}\right\|_{\ell_{2}} \\
& =\left\|G\left(\frac{1}{\sqrt{M}}, \ldots, \frac{1}{\sqrt{M}}\right)^{M}\right\|=1 \\
& \leq \sup _{\|x\|=1} \|_{\ell_{2}} \\
& \leq\|G\| \\
& =\lambda_{1}
\end{aligned}
$$

where $\lambda_{1}$ is the largest eigenvalue of $G$.

The "moreover" part is immediate.

$$
\begin{aligned}
\frac{M}{N} & =\frac{1}{M} F P\left(\left\{\phi_{i}\right\}_{i=1}^{M}\right) \\
& =\frac{1}{M}\left\|\left(\sum_{i=1}^{M}\left|\left\langle\phi_{i}, \phi_{j}\right\rangle\right|^{2}\right)_{j=1}^{M}\right\|_{\ell_{1}} \\
& =\frac{1}{M}\left\|\left(\frac{M}{N}, \ldots, \frac{M}{N}\right)\right\|_{\ell_{1}} \\
& =\frac{1}{M} \sqrt{M}\left\|\left(\frac{M}{N}, \ldots, \frac{M}{N}\right)\right\|_{\ell_{2}}
\end{aligned}
$$




$$
\begin{aligned}
& =\left\|\frac{1}{\sqrt{M}}\left(\frac{M}{N}, \ldots, \frac{M}{N}\right)\right\|_{\ell_{2}} \\
& =\left\|G\left(\frac{1}{\sqrt{M}}, \ldots, \frac{1}{\sqrt{M}}\right)\right\|_{\ell_{2}} \\
& =\|G\| \\
& =\lambda_{1} .
\end{aligned}
$$

Now we will compute the optimal lower Riesz bounds for outer product frames.

Theorem 2.3.5. If $\left\{\phi_{i}\right\}_{i=1}^{M}$ is a unit norm frame for $\mathbb{H}^{N}$, then the lower Riesz bound of $\left\{\phi_{i} \phi_{i}^{*}\right\}_{i=1}^{M}$ is at most $\frac{M(N-1)}{N(M-1)}$.

Proof. Let $G$ be the Gram matrix of $\left\{\phi_{i} \phi_{i}^{*}\right\}_{i=1}^{M}$ with eigenvalues $\lambda_{1} \geq \lambda_{2} \geq \cdots \geq \lambda_{M}$. Then $\operatorname{tr}(G)=M$ gives

$$
\sum_{i=2}^{M} \lambda_{i}=M-\lambda_{1}
$$

Also,

$$
(M-1) \lambda_{M} \leq \sum_{i=2}^{M} \lambda_{i},
$$

and so

$$
\lambda_{M} \leq \frac{\sum_{i=2}^{M} \lambda_{i}}{M-1} .
$$

Finally, we have

$$
\lambda_{M} \leq \frac{M-\lambda_{1}}{M-1} \leq \frac{M-\frac{M}{N}}{M-1}=\frac{M(N-1)}{N(M-1)}
$$


In the next theorem, we see that the above bounds are sharp.

Theorem 2.3.6. Let $\left\{\phi_{i}\right\}_{i=1}^{M}$ be a unit norm equiangular frame for $\mathbb{H}^{N}$ with $M>N$ and let $c:=\left|\left\langle\phi_{i}, \phi_{j}\right\rangle\right|^{2}$ for $i \neq j$. Then $\left\{\phi_{i} \phi_{i}^{*}\right\}_{i=1}^{M}$ is a Riesz sequence whose Gram matrix has two distinct eigenvalues, both of which are non-zero:

$$
\lambda_{1}=1+(M-1) c \text { and } \lambda_{i}=1-c \text { for all } i=2,3, \ldots, M
$$

Moreover, if $\left\{\phi_{i}\right\}_{i=1}^{M}$ is also a tight frame, then $c=\frac{M-N}{N(M-1)}$ and $\left\{\phi_{i} \phi_{i}^{*}\right\}_{i=1}^{M}$ is a Riesz sequence with Riesz bounds $\frac{M(N-1)}{N(M-1)}, \frac{M}{N}$.

Before proving the above result, we need a well-known theorem (see [14]).

Theorem 2.3.7 (Sylvester's Determinant Theorem). Let $S$ and $T$ be matrices of size $M \times N$ and $N \times M$, respectively. Then

$$
\operatorname{det}\left(I_{M}+S T\right)=\operatorname{det}\left(I_{N}+T S\right)
$$

Proof of Theorem 2.3.6. Let $G$ be the Gram matrix for $\left\{\phi_{i} \phi_{i}^{*}\right\}_{i=1}^{M}$. Then

$$
G[i, j]=\left\{\begin{array}{ll}
1 & \text { if } i=j \\
c & \text { otherwise }
\end{array} .\right.
$$

Then we can write $G=(1-c) I_{M}+c 1_{M} 1_{M}^{*}$ and expand using Sylvester's determinant theorem with $S=1_{M}$ and $T=1_{M}^{*}$ :

$$
\begin{aligned}
\operatorname{det}\left((1-c) I_{M}+c 1_{M} 1_{M}^{*}-\lambda I_{M}\right) & =\operatorname{det}\left((1-c-\lambda) I_{M}+c 1_{M} 1_{M}^{*}\right) \\
& =(1-c-\lambda)^{M} \operatorname{det}\left(I_{M}+\frac{c}{1-c-\lambda} 1_{M} 1_{M}^{*}\right) \\
& =(1-c-\lambda)^{M} \operatorname{det}\left(I_{1}+\frac{c}{1-c-\lambda} 1_{M}^{*} 1_{M}\right) \\
& =(1-c-\lambda)^{M-1}(1-c-\lambda+c M) .
\end{aligned}
$$


Setting the above equal to zero and solving for $\lambda$, we get the solutions $\lambda=1-c$, occurring $(M-1)$-times, and $\lambda=1+(M-1) c$, occurring once.

If $c=0$, then $\left\{\phi_{i} \phi_{i}^{*}\right\}_{i=1}^{M}$ are orthonormal, and hence, $\left\{\phi_{i}\right\}_{i=1}^{M}$ are orthonormal as well, which contradicts the assumption that $M>N$. If $c=1$, then $\phi_{i}=\alpha_{i j} \phi_{j}$, with $\left|\alpha_{i j}\right|=1$ for all $i$ and $j$, which contradicts the idea that this is a frame. Hence, $0<c<1$ and the outer products are Riesz.

For the "moreover" part, we compute:

$$
1-c=1-\frac{M-N}{N(M-1)}=\frac{N M-N-M+N}{N(M-1)}=\frac{M(N-1)}{N(M-1)}
$$

and

$$
1+(M-1) c=1+(M-1) \frac{M-N}{N(M-1)}=\frac{N+M-N}{N}=\frac{M}{N} .
$$

We can think of equiangular tight frames as minimizers of the the quantity $B-A$, where $A$ and $B$ are the Riesz bounds of the induced outer products. One problem is that there are few equiangular tight frames. If we want to produce an outer product sequence with arbitrary size and dimension and have predictably good bounds, we cannot use equiangular tight frames. At this time we do not know if there are other frames that achieve the optimal bounds above.

\subsection{Concrete Constructions of Riesz Bases of Outer Products}

Thus far, we have provided no concrete constructions of Riesz outer product sequences. We rectify this with the following examples: 
Example 2.4.1. Let $\left\{e_{i}\right\}_{i=1}^{N}$ be an orthonormal basis for $\mathbb{R}^{N}$ and define $\left\{f_{i j}\right\}$ as follows:

$$
f_{i j}= \begin{cases}e_{i} & \text { if } i=j \\ \frac{1}{\sqrt{2}}\left(e_{i}+e_{j}\right) & \text { if } j>i\end{cases}
$$

for $i=1, \cdots, N$ and $i \leq j$. Then $\left\{E_{i j}\right\}=\left\{f_{i j} f_{i j}^{*}\right\}$ is a Riesz basis for the space of symmetric operators in $\operatorname{sym}\left(\mathbb{R}^{N \times N}\right)$.

Proof. This follows immediately from Proposition 2.2.2.

The following example provides an extension of the above to the complex case. It also provides a second, more intuitive method of verifying that the above example is independent.

Example 2.4.2. Take $\left\{E_{i j}\right\}$ as before and add the following:

$$
E_{i j}^{\prime}=\frac{1}{2}\left(e_{i}+\sqrt{-1} e_{j}\right)\left(e_{i}+\sqrt{-1} e_{j}\right)^{*}
$$

for $j>i$. Then the resulting sequence is Riesz.

Proof. Note that $E_{i j}^{\prime}$ is a matrix with $1^{\prime} s$ in the $(i, i)$ and $(j, j)$ entries and $-\sqrt{-1}$ in the $(i, j)$ entry and $\sqrt{-1}$ in the $(j, i)$ entry. Then we know that $\sum_{i, j} a_{i j} E_{i j}+$ $\sum_{i, j} a_{i j}^{\prime} E_{i j}^{\prime}=0$ if and only if the real and complex parts are 0 . We will do the real part, and the complex part will follow immediately. $E_{i j}$ with $i \neq j$ is the square matrix with 1 's in the $(i, i),(i, j),(j, i)$, and $(j, j)$ entries. Specifically, it is the only element in the sum for which the entries $(i, j)$ and $(j, i)$ could possibly be non-zero. Hence, $a_{i j}=0$ for all $i \neq j$. The remaining terms $E_{i i}$ are orthonormal and, hence, $a_{i i}=0$ for all $i$. Thus the real part is independent, and the complex part follows by the same argument. 
We know that the optimal Riesz bounds for a real Riesz basis of outer products are $(N+1) /(N+2)$ and $(N+1) / 2$. Using unit norm tight frames, we can always achieve the upper bound. The lower bound is then the problem. Here we give a class of unit norm tight frames that produce nice lower bounds as well.

Example 2.4.3. Let $\left\{\phi_{i}\right\}_{i=1}^{N+1}$ be the usual simplex equiangular tight frame for $\mathbb{R}^{N}$. Then consider the outer products

$$
\Phi_{i j}=\left(\frac{\phi_{i}+\phi_{j}}{\left\|\phi_{i}+\phi_{j}\right\|}\right)\left(\frac{\phi_{i}+\phi_{j}}{\left\|\phi_{i}+\phi_{j}\right\|}\right)^{*}
$$

for $j>i$. Then $\Phi_{i j}$ is Riesz provided $N \neq 3$ and has Riesz bounds $\frac{1}{2}$ and $\frac{N+1}{2}$ for $N \geq 7$.

Proof. Barg et al. showed in [3] that the frame

$$
\frac{\phi_{i}+\phi_{j}}{\left\|\phi_{i}+\phi_{j}\right\|}
$$

is a unit norm tight frame. Hence, by Theorem 2.3.4, the upper Riesz bound of the induced outer products is

$$
\frac{N(N+1)}{2} \frac{1}{N}=\frac{N+1}{2}
$$

For the lower bound, we can consider the simplex in $\mathbb{R}^{N}$ as $\left\{\frac{P e_{i}}{\left\|P e_{i}\right\|}\right\}_{i=1}^{N+1}$, where $\left\{e_{i}\right\}_{i=1}^{N+1}$ is an orthonormal basis for $\mathbb{R}^{N+1}, P=I_{N+1}-f f^{*}$, and $f=\frac{1}{\sqrt{N+1}} \sum_{i=1}^{N+1} e_{i}$. Then we have

$$
\begin{aligned}
\phi_{i} & =\frac{P e_{i}}{\left\|P e_{i}\right\|} \\
& =\sqrt{\frac{N+1}{N}}\left(-\frac{1}{N+1}, \ldots,-\frac{1}{N+1}, 1-\frac{1}{N+1},-\frac{1}{N+1}, \ldots,-\frac{1}{N+1}\right)
\end{aligned}
$$


and

$$
\begin{aligned}
\left\langle\phi_{i}, \phi_{j}\right\rangle & =\frac{N+1}{N}\left(\frac{N-1}{(N+1)^{2}}-\frac{2}{N+1}\left(1-\frac{1}{N+1}\right)\right) \\
& =-\frac{1}{N} .
\end{aligned}
$$

Now, $\left\|\phi_{i}+\phi_{j}\right\|^{2}=2 \frac{N-1}{N}$ for $i \neq j$, so we can compute the the Gram matrix of $\left\{\Phi_{i j}\right\}_{i j}$,

$$
G_{\Phi}[i j, k l]=\left\langle\Phi_{i j}, \Phi_{k l}\right\rangle= \begin{cases}1 & \text { if } i=k \text { and } j=l \\ \frac{(N-3)^{2}}{4(N-1)^{2}} & \text { if } i=k \text { or } i=l \text { or } j=k \text { or } j=l \\ \frac{4}{(N-1)^{2}} & \text { if no indices are equal }\end{cases}
$$

Consider the collection of unit norm vectors

$$
E_{i j}=\frac{1}{2}\left(e_{i}+e_{j}\right)\left(e_{i}+e_{j}\right)^{*} \text { for } j>i
$$

where $\left\{e_{i}\right\}_{i=1}^{N+1}$ is an orthonormal basis for $\mathbb{R}^{N+1}$. Now its Gram matrix is

$$
G_{E}[i j, k l]=\left\{\begin{array}{ll}
1 & \text { if } i=k \text { and } j=l \\
\frac{1}{4} & \text { if either } i=k \text { or } i=l \text { or } j=k \text { or } j=l . \\
0 & \text { if no indices are equal }
\end{array} .\right.
$$

This gives us the decomposition

$$
\begin{aligned}
G_{\Phi}= & \left(1-4\left(\frac{(N-3)^{2}}{4(N-1)^{2}}\right)+\frac{1}{(N-1)^{2}}\right) I_{N(N+1) / 2} \\
& +4\left(\frac{(N-3)^{2}}{4(N-1)^{2}}-\frac{4}{(N-1)^{2}}\right) G_{E} \\
& +\frac{4}{(N-1)^{2}} 1_{N(N+1) / 2} 1_{N(N+1) / 2}^{*} .
\end{aligned}
$$

Some inequalities:

$$
\frac{(N-3)^{2}}{4(N-1)^{2}}-\frac{4}{(N-1)^{2}} \geq 0
$$

if $N \geq 7$ and

$$
1-4\left(\frac{(N-3)^{2}}{4(N-1)^{2}}\right)+\frac{12}{(N-1)^{2}}>0
$$


if $N>1$. The matrices

$$
\left(1-\frac{(N-3)^{2}}{(N-1)^{2}}+\frac{12}{(N-1)^{2}}\right) I_{N(N+1) / 2}
$$

and

$$
4\left(\frac{(N-3)^{2}}{4(N-1)^{2}}-\frac{4}{(N-1)^{2}}\right) G_{E}
$$

are positive-definite and $\frac{4}{(N-1)^{2}} 1_{N(N+1) / 2} 1_{N(N+1) / 2}^{*}$ is positive-semidefinite, so

$$
\begin{aligned}
\lambda_{\min }\left[G_{\Phi}\right] \geq & \lambda_{\min }\left[\left(1-\frac{(N-3)^{2}}{(N-1)^{2}}+\frac{12}{(N-1)^{2}}\right) I_{N(N+1) / 2}\right] \\
& +\lambda_{\min }\left[4\left(\frac{(N-3)^{2}}{4(N-1)^{2}}-\frac{4}{(N-1)^{2}}\right) G_{E}\right] \\
& +\lambda_{\min }\left[\frac{4}{(N-1)^{2}} 1_{N(N+1) / 2} 1_{N(N+1) / 2}^{*}\right] \\
= & \left(1-\frac{(N-3)^{2}}{(N-1)^{2}}+\frac{12}{(N-1)^{2}}\right) \\
& +4\left(\frac{(N-3)^{2}}{4(N-1)^{2}}-\frac{4}{(N-1)^{2}}\right) \lambda_{\min }\left[G_{E}\right]+0 .
\end{aligned}
$$

We need to know $\lambda_{\min }\left(G_{E}\right)$.

Let $E_{i j}=\frac{1}{2}\left(e_{i}+e_{j}\right)\left(e_{i}+e_{j}\right)^{*}$. Then

$$
\begin{aligned}
\left\|\sum_{i=1}^{N+1} \sum_{j>i} a_{i j} E_{i j}\right\|^{2} & =\frac{1}{4} \sum_{i=1}^{N+1}\left[\left|\sum_{j>i} a_{i j}+\sum_{j<i} a_{j i}\right|^{2}+2 \sum_{j>i}\left|a_{i j}\right|^{2}\right] \\
& \geq \frac{1}{2} \sum_{j>i}\left|a_{i j}\right|^{2} \\
& =\frac{1}{2}
\end{aligned}
$$

for $a_{i j}$ which square sum to 1 .

Then (2.1) becomes

$$
1-\frac{(N-3)^{2}}{4(N-1)^{2}}+\frac{12}{(N-1)^{2}}+2\left(\frac{(N-3)^{2}}{4(N-1)^{2}}-\frac{4}{(N-1)^{2}}\right)=\frac{(N+1)^{2}}{2(N-1)^{2}} \geq \frac{1}{2}
$$

for $N>1$. 
Because these inequalities only hold for $N \geq 7$, we have computed the lower Riesz bounds for $N=2,3, \ldots, 6$ manually:

\begin{tabular}{|l|l|}
\hline$N$ & lower bound \\
\hline \hline 2 & $3 / 4$ \\
\hline 3 & 0 \\
\hline 4 & $5 / 36$ \\
\hline 5 & $3 / 8$ \\
\hline 6 & $63 / 100$ \\
\hline
\end{tabular}

Remark 2.4.4. When $N=3$ we see another example of the strangeness of this problem. In this case we find that $\Phi_{14}=\Phi_{23}$, thus producing a dependent sequence.

\subsection{Duals of Outer Products}

Lemma 2.5.1. Given a vector $\phi$ in $\mathbb{H}^{N}$ and operators $T_{1}, T_{2}$ acting on $\mathbb{H}^{N}$ with $T_{2}$ self-adjoint, we have $T_{1}\left(\phi \phi^{*}\right) T_{2}=\left(T_{1} \phi\right)\left(T_{2} \phi\right)^{*}$. In particular, $T_{1}\left(\phi \phi^{*}\right)=\left(T_{1} \phi\right) \phi^{*}$.

Proof. We compute for $x \in \mathbb{H}^{N}$

$$
\begin{aligned}
T_{1}\left(\phi \phi^{*}\right) T_{2}(x) & =T_{1}\left\langle T_{2} x, \phi\right\rangle \phi \\
& =T_{1}\left\langle x, T_{2} \phi\right\rangle \phi \\
& =\left(T_{1} \phi\right)\left(T_{2} \phi\right)^{*}(x) .
\end{aligned}
$$

Before the next proposition we need the following definition:

Definition 2.5.2. Let $\left\{\phi_{i}\right\}_{i=1}^{M}$ and $\left\{\psi_{i}\right\}_{i=1}^{M}$ be sequences of vectors in $\mathbb{H}^{N}$. We say $\left\{\phi_{i}\right\}_{i=1}^{M}$ and $\left\{\psi_{i}\right\}_{i=1}^{M}$ are biorthogonal if

$$
\left\langle\phi_{i}, \psi_{j}\right\rangle=\delta_{i j}=\left\{\begin{array}{ll}
1 & \text { if } i=j \\
0 & \text { otherwise }
\end{array} .\right.
$$


Proposition 2.5.3. If $\left\{\phi_{i}\right\}_{i=1}^{M}$ is a Riesz sequence in $\mathbb{H}^{N}$ with biorthogonal vectors $\left\{\tilde{\phi}_{i}\right\}_{i=1}^{M}$, then the biorthogonal vectors for $\left\{\phi_{i} \phi_{i}^{*}\right\}_{i=1}^{M}$ are $\left\{P \tilde{\phi}_{i} \tilde{\phi}_{i}^{*}\right\}_{i=1}^{M}$, where $P$ is the orthogonal projection onto the span of $\left\{\phi_{i} \phi_{i}^{*}\right\}_{i=1}^{M}$.

Proof. We compute

$$
\left\langle\phi_{i} \phi_{i}^{*}, P \tilde{\phi}_{j} \tilde{\phi}_{j}^{*}\right\rangle_{F}=\left\langle P \phi_{i} \phi_{i}^{*}, \tilde{\phi}_{j} \tilde{\phi}_{j}^{*}\right\rangle_{F}=\left|\left\langle\phi_{i}, \tilde{\phi}_{j}\right\rangle\right|^{2}=\delta_{i j}
$$

So the vectors $\left\{P \tilde{\phi}_{i} \tilde{\phi}_{i}^{*}\right\}_{i=1}^{M}$ are biorthogonal to $\left\{\phi_{i} \phi_{i}^{*}\right\}_{i=1}^{M}$.

Remark 2.5.4. Projecting is necessary in the above proposition. For example, assume $\left\{\phi_{1}, \phi_{2}\right\}$ is a non-orthogonal Riesz basis for $\mathbb{R}^{2}$. Then $\tilde{\phi}_{1} \perp \phi_{2}$, so take any $\psi_{1} \perp \phi_{2}$ with norm 1 and scale $\tilde{\phi}_{1}$ so that $\left\langle\phi_{1}, \tilde{\phi}_{1}\right\rangle=1$ i.e. $\tilde{\phi}_{1}=\frac{1}{\left\langle\psi_{1}, \phi_{1}\right\rangle} \psi_{1}$. Then the Gram matrix of the induced outer products of $\left\{\phi_{1}, \phi_{2}, \tilde{\phi}_{1}\right\}$ is

$$
\left[\begin{array}{ccc}
1 & \left|\left\langle\phi_{1}, \phi_{2}\right\rangle\right|^{2} & 1 \\
\left|\left\langle\phi_{1}, \phi_{2}\right\rangle\right|^{2} & 1 & 0 \\
1 & 0 & 1
\end{array}\right]
$$

which has determinant $-\left|\left\langle\phi_{1}, \phi_{2}\right\rangle\right|^{4}$. Because we have chosen $\phi_{1} \not \subset \phi_{2}$ this matrix is invertible. Hence, these outer products are Riesz. But then $\tilde{\phi}_{1} \tilde{\phi}_{1}^{*}$ is not in the span of the other two, which means that the projections are necessary.

\subsection{Outer Cross-Products of Frames}

We now turn to a generalization of what we have done so far. Rather than considering $\left\{\phi_{i} \phi_{i}^{*}\right\}_{i=1}^{M}$, we will examine the set of all rank-one matrices obtainable through outer products. Specifically, for collections of vectors $\left\{\phi_{i}\right\}_{i=1}^{M}$ and $\left\{\psi_{i}\right\}_{i=1}^{L}$ in $\mathbb{H}^{N}$, we will consider the collection $\left\{\phi_{i} \psi_{j}^{*}\right\}_{i=1, j=1}^{M}$. One immediate difference is that these outer products are no longer symmetric, even if the original sequences are equal. As such, the ambient space is no longer the space of self-adjoint matrices; instead, it is the 
space of all matrices of size $N \times N$. Another interesting aspect of such outer products is that the Gram matrix takes the form of another famous product in matrix theory.

Definition 2.6.1. Let $S$ and $T$ be matrices of arbitrary size. The Kronecker product of $S$ and $T$ is the block matrix

$$
S \otimes T=[S[i, j] T]_{i j}
$$

Lemma 2.6.2. Let $G_{\phi}$ and $G_{\psi}$ be the Gram matrices of $\left\{\phi_{i}\right\}_{i=1}^{M}$ and $\left\{\psi_{j}\right\}_{j=1}^{L}$, respectively. The Gram matrix of $\left\{\phi_{i} \psi_{j}^{*}\right\}_{i=1, j=1}^{M, L}$ is $G_{\phi} \otimes G_{\psi}^{T}$.

Proof. First note that

$$
\left\langle\phi_{i} \psi_{j}^{*}, \phi_{k} \psi_{l}^{*}\right\rangle=\left\langle\phi_{i}, \phi_{k}\right\rangle\left\langle\psi_{l}, \psi_{j}\right\rangle
$$

which means that if we arrange our outer products

$$
\left\{\phi_{1} \psi_{1}^{*}, \phi_{1} \psi_{2}^{*}, \ldots, \phi_{1} \psi_{M}^{*}, \phi_{2} \psi_{1}^{*}, \ldots, \phi_{M} \psi_{M}^{*}\right\}
$$

then the Gram matrix of this collection of vectors is

$$
\left[\left\langle\phi_{i}, \phi_{k}\right\rangle\left\langle\psi_{l}, \psi_{j}\right\rangle\right]_{i j, k l}=G_{\phi} \otimes G_{\psi}^{T}
$$

Now we are able to take advantage of another well-known result from matrix theory (see $[15])$.

Theorem 2.6.3. Let $S$ and $T$ be square matrices with eigenvalues $\left\{\lambda_{i}\right\}_{i=1}^{M}$ and $\left\{\nu_{i}\right\}_{i=1}^{L}$, respectively. The eigenvalues of $S \otimes T$ are $\left\{\lambda_{i} \nu_{j}\right\}_{i=1, j=1}^{M, L}$.

We now come to our main result on outer cross-products. 
Theorem 2.6.4. If $\left\{\phi_{i}\right\}_{i=1}^{M}$ and $\left\{\psi_{j}\right\}_{j=1}^{L}$ are collections of vectors in $\mathbb{H}^{N}$ that are

1. frames with frame bounds $A, B$ and $C, D$, respectively, then $\left\{\phi_{i} \psi_{j}^{*}\right\}_{i=1, j=1}^{M, L}$ is a frame for $\mathbb{H}^{N \times N}$, with frame bounds $A C, B D$.

2. Riesz sequences with Riesz bounds $A, B$ and $C, D$ then $\left\{\phi_{i} \psi_{j}^{*}\right\}_{i=1, j=1}^{M, L}$ is Riesz sequence for $\mathbb{H}^{N \times N}$, with Riesz bounds $A C, B D$.

Proof. Let $G_{\phi}$ and $G_{\psi}$ be the Gram matrices of $\left\{\phi_{i}\right\}_{i=1}^{M}$ and $\left\{\psi_{j}\right\}_{j=1}^{L}$, respectively. Further, suppose that $G_{\phi}$ has eigenvalues $\left\{\lambda_{i}\right\}_{i=1}^{M}$ and $G_{\psi}$ has eigenvalues $\left\{\nu_{i}\right\}_{i=1}^{L}$. Assume that $\lambda_{1} \geq \lambda_{2} \geq \cdots \geq \lambda_{M}$ and $\nu_{1} \geq \nu_{2} \geq \cdots \geq \nu_{L}$.

If $\left\{\phi_{i}\right\}_{i=1}^{M}$ is a frame with frame bounds $A$ and $B$, then $A=\lambda_{N}$ and $B=\lambda_{1}$. Likewise, if $\left\{\psi_{j}\right\}_{j=1}^{L}$ is a frame with frame bounds $C$ and $D$, then $C=\nu_{N}$ and $D=\nu_{1}$. Then $G_{\phi} \otimes G_{\psi}^{T}$ has $N^{2}$ strictly positive eigenvalues, so $\left\{\phi_{i} \psi_{j}^{*}\right\}_{i=1, j=1}^{M, L}$ is a frame for $\mathbb{H}^{N \times N}$. The frame bounds are the largest and smallest non-zero eigenvalues of $G_{\phi} \otimes G_{\psi}^{T}$, which are $B D$ and $A C$, respectively.

If, on the other hand, $\left\{\phi_{i}\right\}_{i=1}^{M}$ and $\left\{\psi_{j}\right\}_{j=1}^{L}$ are Riesz sequences, then $\lambda_{M}>0$ and $\nu_{L}>0$, so $\lambda_{i} \nu_{j}>0$ for all $i, j$. Hence, $\left\{\phi_{i} \psi_{j}^{*}\right\}_{i=1, j=1}^{M, L}$ is Riesz with Riesz bounds $B D$ and $A C$.

In the case of symmetric matrices (see Proposition 2.5.3), to find the dual functionals of a Riesz sequence of outer products, we had to project the desired functionals onto the span of the outer products. Now we show that this assumption is not necessary in the general case of outer cross-products.

Theorem 2.6.5. If $\left\{\phi_{i}\right\}_{i=1}^{N}$ and $\left\{\psi_{i}\right\}_{i=1}^{N}$ are Riesz bases in $\mathbb{H}^{N}$ with dual Riesz bases $\left\{\tilde{\phi}_{i}\right\}_{i=1}^{N}$ and $\left\{\tilde{\psi}_{i}\right\}_{i=1}^{N}$, respectively, then $\left\{\phi_{i} \psi_{j}^{*}\right\}_{i, j=1}^{N}$ is a Riesz basis for $\mathbb{H}^{N \times N}$, with 
dual basis $\left\{\tilde{\phi}_{i} \tilde{\psi}_{j}^{*}\right\}_{i, j=1}^{N}$.

Proof. We compute

$$
\left\langle\tilde{\phi}_{i} \tilde{\psi}_{j}^{*}, \phi_{l} \psi_{k}^{*}\right\rangle_{F}=\left\langle\tilde{\phi}_{i}, \phi_{l}\right\rangle\left\langle\psi_{k}, \tilde{\psi}_{j}\right\rangle=\left\{\begin{array}{ll}
1 & \text { if } l=i \text { and } j=k \\
0 & \text { otherwise }
\end{array} .\right.
$$

\subsection{Topological Properties of Independent Outer Product Sequences}

In this section, we will consider the family of unit norm frames with cardinality $M \leq$ $\operatorname{dim} \operatorname{sym}\left(\mathbb{H}^{N \times N}\right)$. We see that we can identify this family with the topological space $\bigotimes_{i=1}^{M} S^{N-1}$, where $S^{N-1}$ is the unit sphere in $\mathbb{H}^{N}$. We will use the standard metric for frames, $d(\Phi, \Psi)=\sqrt{\sum_{i=1}^{M}\left\|\phi_{i}-\psi_{i}\right\|^{2}}$, which is compatible with the subspace topology

of the Euclidean topology with regards to $\bigotimes_{i=1}^{M} S^{N-1}$. Such results are often proved in frame theory using algebraic geometry, which might give a slightly stronger result that the unit norm $M$-element frames that produce independent outer products form an open dense set in the Zariski topology in the family of all unit norm $M$-element frames. Instead, we offer a direct, analytic construction for the density of the frames giving independent outer products.

Lemma 2.7.1. If $\left\{\phi_{i}\right\}_{i=1}^{N}$ is a Riesz sequence in $\mathbb{H}^{N}$ with Riesz bounds $A, B$ and

$$
\sum_{i=1}^{N}\left\|\phi_{i}-\psi_{i}\right\|^{2}<\varepsilon^{2}<A
$$

then $\left\{\psi_{i}\right\}_{i=1}^{N}$ is Riesz with Riesz bounds $(\sqrt{A}-\varepsilon)^{2},(\sqrt{B}+\varepsilon)^{2}$.

Proof. For any $\left\{a_{i}\right\}_{i=1}^{N}$ we compute

$$
\left\|\sum_{i=1}^{N} a_{i} \psi_{i}\right\| \leq\left\|\sum_{i=1}^{N} a_{i} \phi_{i}\right\|+\left\|\sum_{i=1}^{N} a_{i}\left(\psi_{i}-\phi_{i}\right)\right\|
$$




$$
\begin{aligned}
& \leq B^{1 / 2}\left(\sum_{i=1}^{N}\left|a_{i}\right|^{2}\right)^{1 / 2}+\sum_{i=1}^{N}\left|a_{i}\right|\left\|\psi_{i}-\phi_{i}\right\| \\
& \leq B^{1 / 2}\left(\sum_{i=1}^{N}\left|a_{i}\right|^{2}\right)^{1 / 2}+\left(\sum_{i=1}^{N}\left|a_{i}\right|^{2}\right)^{1 / 2}\left(\sum_{i=1}^{N}\left\|\psi_{i}-\phi_{i}\right\|^{2}\right)^{1 / 2} \\
& \leq\left(B^{1 / 2}+\varepsilon\right)\left(\sum_{i=1}^{N}\left|a_{i}\right|^{2}\right)^{1 / 2} .
\end{aligned}
$$

The stated upper Riesz bound is immediate from here. The lower Riesz bound follows similarly.

Lemma 2.7.2. If $\|\phi\|=\|\psi\|=1$, then

$$
\left\|\phi \phi^{*}-\psi \psi^{*}\right\|_{F}^{2} \leq 2\|\phi-\psi\|^{2} .
$$

Proof. We compute

$$
\begin{aligned}
\left\|\phi \phi^{*}-\psi \psi^{*}\right\|_{F}^{2} & =\left\|\phi \phi^{*}\right\|_{F}^{2}+\left\|\psi \psi^{*}\right\|_{F}^{2}-2 \operatorname{Re}\left\langle\phi \phi^{*}, \psi \psi^{*}\right\rangle_{F} \\
& =1+1-2|\langle\phi, \psi\rangle|^{2} \\
& =2\left(1-|\langle\phi, \psi\rangle|^{2}\right) \\
& =2(1-|\langle\phi, \psi\rangle|)(1+|\langle\phi, \psi\rangle|) \\
& =(2-2|\langle\phi, \psi\rangle|)(1+|\langle\phi, \psi\rangle|) \\
& \leq(2-2 \operatorname{Re}\langle\phi, \psi\rangle)(1+|\langle\phi, \psi\rangle|) \\
& =\left(\|\phi\|^{2}+\|\psi\|^{2}-2 \operatorname{Re}\langle\phi, \psi\rangle\right)(1+|\langle\phi, \psi\rangle|) \\
& =\|\phi-\psi\|^{2}(1+|\langle\phi, \psi\rangle|) \\
& \leq 2\|\phi-\psi\|^{2} .
\end{aligned}
$$


Proposition 2.7.3. Assume that $\left\{\phi_{i}\right\}_{i=1}^{M}$ are unit norm vectors in $\mathbb{H}^{N}$ such that $\left\{\phi_{i} \phi_{i}^{*}\right\}_{i=1}^{M}$ is a Riesz sequence having Riesz bounds $A, B$. Given $0<\varepsilon<A / 2$, choose a unit norm set of vectors $\left\{\psi_{i}\right\}_{i=1}^{M}$ so that

$$
\sum_{i=1}^{M}\left\|\phi_{i}-\psi_{i}\right\|^{2}<\varepsilon<\frac{A}{2}
$$

Then $\left\{\psi_{i} \psi_{i}^{*}\right\}_{i=1}^{M}$ is Riesz with Riesz bounds

$$
(\sqrt{A}-\sqrt{2 \varepsilon})^{2} \text { and }(\sqrt{B}+\sqrt{2 \varepsilon})^{2}
$$

Proof. Assume the hypotheses. It follows from our Lemma 2.7.2 that

$$
\sum_{i=1}^{M}\left\|\phi_{i} \phi_{i}^{*}-\psi_{i} \psi_{i}^{*}\right\|_{F}^{2} \leq 2 \sum_{i=1}^{M}\left\|\phi_{i}-\psi_{i}\right\|^{2}<2 \varepsilon
$$

Now by Lemma 2.7.1. we have that $\left\{\psi_{i} \psi_{i}^{*}\right\}_{i=1}^{M}$ is Riesz with Riesz bounds

$$
(\sqrt{A}-\sqrt{2 \varepsilon})^{2},(\sqrt{B}+\sqrt{2 \varepsilon})^{2}
$$

The above proposition says that the set of frames with cardinality $M \leq \operatorname{dim} \operatorname{sym}\left(\mathbb{H}^{N \times N}\right)$ is open in $\bigotimes_{i=1}^{M} S^{N-1}$. In the remainder of this section, we will show that this set is also dense. While other authors have studied the density of outer products in terms of commutative algebra (see [5]), here we show this fact constructively and quantitatively using only standard analytic and Euclidean topological notions.

Lemma 2.7.4. Let $S$ be an invertible operator, and suppose $\left\{\phi_{i}\right\}_{i=1}^{M}$ are vectors in $\mathbb{H}^{N}$. Then $\left\{\phi_{i} \phi_{i}^{*}\right\}_{i=1}^{M}$ is independent if and only if $\left\{S \phi_{i}\left(S \phi_{i}\right)^{*}\right\}_{i=1}^{M}$ is independent. 
Proof. Let $\left\{a_{i}\right\}_{i=1}^{M}$ be scalars, not all zero. We have

$$
0=\sum_{i=1}^{M} a_{i} \phi_{i} \phi_{i}^{*}
$$

if and only if

$$
0=S\left(\sum_{i=1}^{M} a_{i} \phi_{i} \phi_{i}^{*}\right) S^{*}=\sum_{i=1}^{M} a_{i}\left(S \phi_{i}\right)\left(S \phi_{i}\right)^{*} .
$$

Now we construct a large family of bases of outer products.

Lemma 2.7.5. Given a unit norm vector $\psi \in \mathbb{H}^{N}$ and $\varepsilon>0$, there is a unit norm basis for $\operatorname{sym}\left(\mathbb{H}^{N \times N}\right)$ consisting of outer products $\left\{\phi_{i} \phi_{i}^{*}\right\}_{i=1}^{d}$ with $d=\operatorname{dim} \operatorname{sym}\left(\mathbb{H}^{N \times N}\right)$, such that $\left\|\phi_{i}-\psi\right\|^{2}<\varepsilon$ for all $i=1, \ldots, d$.

Proof. First, we will assume that we have a unit norm basis $\left\{\psi_{i} \psi_{i}^{*}\right\}_{i=1}^{d}$ of $\operatorname{sym}\left(\mathbb{H}^{N \times N}\right)$ with $\left\langle\psi, \psi_{i}\right\rangle>0$ for all $i$ and $\psi=e_{1}$ for an orthonormal basis $\left\{e_{j}\right\}_{j=1}^{N}$ of $\mathbb{H}^{N}$. We can see that such a basis exists by a unitary transformation of Example 2.4.1 or Example 2.4.2. Choose $\delta>0$ with the following property: if

$$
S=\operatorname{diag}(1, \delta, \delta, \ldots, \delta)
$$

then for all $i=1,2, \ldots, d$, we have

$$
\sum_{j=2}^{N}\left|S \psi_{i}(j)\right|^{2}=\delta^{2} \sum_{j=2}^{N}\left|\psi_{i}(j)\right|^{2} \leq \frac{\varepsilon}{2}\left|\psi_{i}(1)\right|^{2} \leq \frac{\varepsilon}{2}\left\|S \psi_{i}\right\|^{2} .
$$

Let

$$
\phi_{i}=\frac{S \psi_{i}}{\left\|S \psi_{i}\right\|} \text { for all } i=1,2, \ldots, d
$$

and observe that $\left\|\phi_{i}\right\|=1$ and Equation 2.2 together imply

$$
\phi_{i}(1) \geq 1-\frac{\varepsilon}{2}
$$


Now we compute for all $i=1,2, \ldots, d$

$$
\left\|\psi-\phi_{i}\right\|^{2}=\left|1-\phi_{i}(1)\right|^{2}+\sum_{j=2}^{N}\left|\phi_{i}(j)\right|^{2} \leq \epsilon
$$

Because $\left\{\psi_{i} \psi_{i}^{*}\right\}_{i=1}^{d}$ is linearly independent, then by Lemma 2.7.4, the $\left\{\phi_{i} \phi_{i}^{*}\right\}_{i=1}^{d}$ are also independent.

For the general case, given $\psi$ and $\left\{\psi_{i}\right\}_{i=1}^{d}$ with independent outer products, choose a vector $\phi$ so that $\left\langle\phi, \psi_{i}\right\rangle \neq 0$ for all $i=1,2, \ldots, d$. By replacing $\phi$ by $c_{i} \phi$ with $\left|c_{i}\right|=1$ if necessary, we can assume these inner products are all strictly positive. By the above, we can find $\left\{\phi_{i}\right\}_{i=1}^{d}$ with their outer products independent and

$$
\left\|\phi-\phi_{i}\right\|^{2}<\varepsilon
$$

Choose a unitary operator $U$ so that $U \phi=\psi$, and we have

$$
\left\|\psi-U \phi_{i}\right\|^{2}=\left\|U \phi-U \phi_{i}\right\|^{2}=\left\|\phi-\phi_{i}\right\|^{2}<\varepsilon
$$

This completes the proof.

With the above lemmas, we are ready to prove the following:

Theorem 2.7.6. The set of all frames $\left\{\phi_{i}\right\}_{i=1}^{M}$ with $M \leq \operatorname{dim} \operatorname{sym}\left(\mathbb{H}^{N}\right)$ that produce independent outer products is open and dense in the family of $M$-element frames.

Proof. This set was already shown to be open by Proposition 2.7.3. All that remains to be shown is that this set is also dense. Let $\phi_{1}^{\prime}=\phi_{1}$, and proceed by induction. Assume that we have a collection of vectors $\left\{\phi_{i}^{\prime}\right\}_{i=1}^{M_{0}}$ such that $\left\|\phi_{i}^{\prime}-\phi_{i}\right\|<\varepsilon / M$ for all $i=1, \ldots, M_{0}$ and $\left\{\phi_{i}^{\prime}\left(\phi_{i}^{\prime}\right)^{*}\right\}_{i=1}^{M_{0}}$ is independent. Then by Lemma 2.7.5, there exists a unit norm basis $\left\{\psi_{i}\right\}_{i=1}^{\operatorname{dim} \operatorname{sym}\left(\mathbb{H}^{N \times N}\right)}$ such that $\left\|\phi_{M_{0}+1}-\psi_{i}\right\|<\varepsilon / M$ for all $i$. 
Since $\left\{\psi_{i} \psi_{i}^{*}\right\}_{i=1}^{\operatorname{dim} \operatorname{sym}\left(\mathbb{H}^{N \times N}\right)}$ is a basis, we can choose $\phi_{M_{0}+1}^{\prime}=\psi_{k}$ such that $\psi_{k} \psi_{k}^{*} \notin$ $\operatorname{span}\left(\left\{\phi_{i}^{\prime}\left(\phi_{i}^{\prime}\right)^{*}\right\}_{i=1}^{M_{0}}\right)$. Then the set $\left\{\phi_{i}^{\prime}\right\}_{i=1}^{M_{0}+1}$ induces independent outer products with $\left\|\phi_{i}^{\prime}-\phi_{i}\right\|<\varepsilon / M$ for all $i$. By induction, we have obtained a set $\left\{\phi_{i}^{\prime}\right\}_{i=1}^{M}$ such that

$$
\sum_{i=1}^{M}\left\|\phi_{i}^{\prime}-\phi_{i}\right\|<\varepsilon
$$

which induces independent outer products.

\subsection{Rank of Bordered Positive Semidefinite Matri- ces and Dependent Outer Product Sequences}

It should be emphasized that we are interested in classifying dependent sets; as we have seen in the previous section, these are far less common than independent sets.

We present a result on the preservation of rank in the process known as bordering. Its proof uses a well-known theorem (see [14] for example).

Theorem 2.8.1 (Determinant of Schur Complement). Let

$$
M=\left[\begin{array}{ll}
A & B \\
C & D
\end{array}\right]
$$

with $D$ invertible. Then

$$
\operatorname{det}(M)=\operatorname{det}(D) \operatorname{det}\left(A-B D^{-1} C\right)
$$

Lemma 2.8.2. Let $A$ be positive definite with eigenvectors $\left\{e_{1}, e_{2}, \ldots, e_{N}\right\}$ and corresponding eigenvalues $\lambda_{1}, \ldots, \lambda_{N}$. Then

$$
B=\left[\begin{array}{ll}
A & v \\
v^{*} & 1
\end{array}\right]
$$

is non-singular if and only if

$$
\sum_{i=1}^{N} \frac{\left|\left\langle v, e_{i}\right\rangle\right|^{2}}{\lambda_{i}} \neq 1
$$


Proof. We compute using Theorem 2.8.1 and Theorem 2.3.7

$$
\begin{aligned}
\operatorname{det}(B) & =\operatorname{det}\left(A-v v^{*}\right) \\
& =\operatorname{det}\left(A A^{-1}\right) \operatorname{det}\left(A-v v^{*}\right) \\
& =\operatorname{det}(A) \operatorname{det}\left(I-A^{-1} v v^{*}\right) \\
& =\operatorname{det}(A) \operatorname{det}\left(1-v^{*} A^{-1} v\right) .
\end{aligned}
$$

Now $\operatorname{det}(A) \operatorname{det}\left(1-v^{*} A^{-1} v\right)=0$ if and only if $\left\langle A^{-1} v, v\right\rangle=1$. Then $A^{-1} v=$ $\sum_{i=1}^{M} \frac{\left\langle v, e_{i}\right\rangle}{\lambda_{i}} e_{i}$ and $v=\sum_{i=1}^{M}\left\langle v, e_{i}\right\rangle e_{i}$ so $\left\langle A^{-1} v, v\right\rangle=\sum_{i=1}^{M} \frac{\left|\left\langle v, e_{i}\right\rangle\right|^{2}}{\lambda_{i}}$.

Now we extend the above to positive semidefinite matrices.

Theorem 2.8.3. Let $A$ be positive semidefinite with eigenvalues $\lambda_{1} \geq \cdots \geq \lambda_{N}$. Let $I_{+}=\left\{i: \lambda_{i}>0\right\}$ and $V_{+}=\operatorname{span}\left\{e_{i}: i \in I_{+}\right\}$. We have

$$
\operatorname{rank}\left(\left[\begin{array}{cc}
A & v \\
v^{*} & 1
\end{array}\right]\right)=\operatorname{rank}(A)
$$

if and only if

$$
\sum_{i \in I_{+}} \frac{\left|\left\langle v, e_{i}\right\rangle\right|^{2}}{\lambda_{i}}=1
$$

and $v \in V_{+}$.

Proof. Without loss of generality, assume that $A$ is diagonal. Let

$$
B=\left[\begin{array}{cc}
A & v \\
v^{*} & 1
\end{array}\right]
$$

and let $B\left(I_{+}\right)$denote the matrix obtained by deleting rows and columns whose indices are in $I_{+}^{c}$. Note that if $v \in V_{+}$, then $v \perp e_{i}$ for all $i \in I_{+}^{c}$. Clearly, if $v \in V_{+}$, then $\operatorname{rank}\left(B\left(I_{+}\right)\right)=\operatorname{rank}(B)$, and we can apply Lemma 2.8.2 to $B\left(I_{+}\right)$to obtain the desired result. 
For the converse, we only need the following: if $\operatorname{rank}(B)=\operatorname{rank}(A)$, then $v \in V_{+}$. First, note that $\operatorname{rank}(B) \geq \operatorname{rank}(A)$ for all $v \in \mathbb{H}^{N}$. Suppose that $v \not L e_{i}$ for some $i$ with $\lambda_{i}=0$. Denote the $i^{t h}$ row of $B$ as $B_{i}$. Then $B_{i}=\left(\lambda_{i} e_{i}^{*}, v(i)\right)$ for $i=1, \ldots, N$. Clearly, $B_{M+1}=\left(v^{*}, 1\right) \notin \operatorname{span}\left\{B_{i}: i \leq N\right\}$ and $\operatorname{rank}(B)>\operatorname{rank}(A)$. Thus, if $\operatorname{rank}(B)=\operatorname{rank}(A)$, then $v \in V^{+}$.

What we have shown is the following: if $B=\left[\begin{array}{ll}A & v \\ v^{*} & 1\end{array}\right]$ with $A$ positive semidefinite, then $\operatorname{rank}(B)=\operatorname{rank}(A)$ if and only if $v=\sum_{i \in I_{+}} a_{i} \sqrt{\lambda_{i}} e_{i}$ with $\sum_{i \in I_{+}}\left|a_{i}\right|^{2}=1$. We will now apply the above to the Gram matrix of an independent outer product sequence.

Corollary 2.8.4. Let $\left\{\phi_{i} \phi_{i}^{*}\right\}_{i=1}^{M}$ be a Riesz sequence in $\mathbb{H}^{N \times N}$ with Gram matrix $G$. Suppose $G$ has eigenvalues $\lambda_{1}, \ldots, \lambda_{M}$ with corresponding eigenvectors $e_{1}, \ldots, e_{N}$. For any unit norm $\psi \in \mathbb{H}^{N},\left\{\phi_{i} \phi_{i}^{*}\right\}_{i=1}^{M} \cup\left\{\psi \psi^{*}\right\}$ is dependent if and only if

$$
\left(\left|\left\langle\phi_{M}, \psi\right\rangle\right|^{2}, \ldots,\left|\left\langle\phi_{M}, \psi\right\rangle\right|^{2}\right)^{T}=\sum_{i=1}^{M} a_{i} \sqrt{\lambda_{i}} e_{i}
$$

for some $\left(a_{i}\right)_{i=1}^{M} \in \mathbb{R}^{M}$ with $\sum_{i=1}^{M}\left|a_{i}\right|^{2}=1$.

\subsection{The Spark of Induced Outer Product Sequences}

The results presented here were developed jointly with Peter Casazza and Victor Kaftal. Full spark frames are studied in [1], in which the authors show that the collection of full spark frames is open and dense in the $\bigotimes_{i=1}^{M} S^{N-1}$. In this section, we investigate the topological properties of the set of frames that produce full spark outer products. We start with a very simple observation that follows immediately from Corollary 2.1.6. 
Proposition 2.9.1. Let $\left\{\phi_{i}\right\}_{i=1}^{M}$ be full spark in $\mathbb{H}^{N}$. Then Spark $\left(\left\{\phi_{i} \phi_{i}^{*}\right\}_{i=1}^{M}\right) \geq N+1$.

Proof. Let $\left\{\phi_{i}\right\}_{i=1}^{M}$ be a full spark frame for $\mathbb{H}^{N}$. Then $\left\{\phi_{i}\right\}_{i \in \Lambda}$ is linearly independent for each $\Lambda \subset[M]$ with $|\Lambda|=M$. Hence, $\left\{\phi_{i} \phi_{i}^{*}\right\}_{i \in \Lambda}$ is independent by Corollary 2.1.6. Thus, the smallest linearly dependent subset of $\left\{\phi_{i} \phi_{i}^{*}\right\}_{i=1}^{M}$ has cardinality at least $N+1$.

Next, we will present a theorem from [1]. This theorem, combined with the simple proposition above, gives us a trivial result on the spark of induced outer product frames.

Theorem 2.9.2. The collection of full spark frames in $\bigotimes_{i=1}^{M} S^{N-1}$ is open and dense.

We immediately get the following:

Proposition 2.9.3. The collection of frames $\left\{\phi_{i}\right\}_{i=1}^{M} \in \bigotimes_{i=1}^{M} S^{N-1}$, such that

$$
\operatorname{Spark}\left(\left\{\phi_{i} \phi_{i}^{*}\right\}_{i=1}^{M}\right) \geq N+1
$$

is open and dense.

Though the above discussion quickly and easily gives us a result for the spark of induced outer product frames, spark $N+1$ is hardly exciting. In the remainder of this section, we will present a series of lemme that will give us a more satisfying result for frames that produce full spark outer products.

Lemma 2.9.4. Let $\left\{\phi_{i}\right\}_{i=1}^{M} \subset S^{N-1}$ be such that $\left\{\phi_{i} \phi_{i}^{*}\right\}_{i=1}^{M} \subset \mathbb{H}^{N \times N}$ is full spark. Then the set

$$
\left\{\phi \in S^{N-1}:\left\{\phi_{i} \phi_{i}^{*}\right\}_{i=1}^{M} \cup\left\{\phi \phi^{*}\right\} \text { is full spark }\right\}
$$

is open and dense in $S^{N-1}$. 
Proof. Define

$$
T: S^{N-1} \rightarrow \Omega, \quad \text { by } \quad T: \phi \mapsto \phi \phi^{*}
$$

$T$ is continuous. Let $d=\operatorname{dim} \operatorname{sym}\left(\mathbb{H}^{N \times N}\right)$, and define the following family of subspaces,

$$
\omega_{j}=\operatorname{span}\left\{\phi_{i} \phi_{i}^{*}\right\}_{i \in \Lambda_{j}}
$$

where $\Lambda_{j} \in\left\{\Lambda_{j} \subset[M]:\left|\Lambda_{j}\right|=d-1\right\}$. Note that $\Omega \backslash \omega_{j}$ is open in $\Omega$ since $\omega_{j}$ is closed. We claim that $\Omega \backslash \omega_{j}$ is dense in $\Omega$. Indeed, let $\phi \phi^{*} \in \Omega$. By Lemma 2.7.5 and Lemma 2.7.2, there exists a basis $\left\{\psi_{i} \psi_{i}^{*}\right\}_{i=1}^{d}$ such that $\left\|\phi \phi^{*}-\psi_{i} \psi_{i}^{*}\right\|<\varepsilon$ for all $i$. Since $\left\{\psi_{i} \psi_{i}^{*}\right\}_{i=1}^{d}$ is a basis, at least one element must belong to $\Omega \backslash \omega_{j}$. Thus $\Omega \backslash \omega_{j}$ is open and dense in $\Omega$. Hence,

$$
T^{-1}\left(\Omega \backslash \omega_{j}\right)
$$

is open and dense in $S^{N-1}$. Finally,

$$
\bigcap_{j} T^{-1}\left(\Omega \backslash \omega_{j}\right)
$$

is open and dense in $S^{N-1}$ by the Baire category theorem. Thus, the set of

$$
\phi \in \bigcap_{j} T^{-1}\left(\Omega \backslash \omega_{j}\right)
$$

such that $\{T \phi\} \cup\left\{\phi_{i} \phi_{i}^{*}\right\}_{i \in \Lambda_{j}}$ has span of dimension $d$ and is linearly independent for each $j$ is open and dense in $S^{N-1}$.

Now we give a somewhat constructive proof that the collection of frames that produce full spark outer products is dense. 
Lemma 2.9.5. The set of frames in $\bigotimes_{i=1}^{M} S^{N-1}$ which produce collections of full spark outer products is dense in $\bigotimes_{i=1}^{M} S^{N-1}$.

Proof. Fix $\varepsilon>0$ and let $\left\{\phi_{i}\right\}_{i=1}^{M}$ be unit norm with $M \geq d$ where $d$ is the dimension of the symmetric matrices. First, we construct a linearly independent set using Lemma 2.7.5. By Lemma 2.7.5, we know that there exists $\left\{\psi_{i}\right\}_{i=1}^{d}$ such that $\left\{\psi_{i} \psi_{i}^{*}\right\}_{i=1}^{d}$ are independent and $\left\|\psi_{i}-\phi_{i}\right\|<\varepsilon / M$ for all $i$. Then by definition, $\left\{\psi_{i} \psi_{i}^{*}\right\}_{i=1}^{d}$ is trivially full spark. Then using Lemma 2.9.4, we will add one vector at a time, replacing if necessary. Suppose that for some $d \leq M_{0} \leq M,\left\{\psi_{i} \psi_{i}^{*}\right\}_{i=1}^{M_{0}}$ is full spark but $\left\{\psi_{i} \psi_{i}^{*}\right\}_{i=1}^{M_{0}} \cup\left\{\phi_{M_{0}+1} \phi_{M_{0}+1}^{*}\right\}$ is not. By Lemma 2.9.4, there is a $\psi_{M_{0}+1}$ such that $\left\{\psi_{i} \psi_{i}^{*}\right\}_{i=1}^{M_{0}} \cup\left\{\psi_{M_{0}+1} \psi_{M_{0}+1}^{*}\right\}$ is full spark and $\left\|\psi_{M_{0}+1}-\phi_{M_{0}+1}\right\|<\varepsilon / M$. Do this for each remaining vector $\phi_{i}$ and the resulting collection $\left\{\psi_{i} \psi_{i}^{*}\right\}_{i=1}^{M}$ is full spark. Furthermore, we have

$$
\sum_{i=1}^{M}\left\|\phi_{i}-\psi_{i}\right\|<\varepsilon
$$

Next, we see that the collection of frames that produce full spark outer products is open.

Lemma 2.9.6. Let $\Phi=\left\{\phi_{i} \phi_{i}^{*}\right\}_{i=1}^{M}$ be full spark. There exists $\varepsilon>0$ such that for all

$$
\begin{aligned}
\Psi= & \left\{\psi_{i} \psi_{i}^{*}\right\}_{i=1}^{M} \text { with } \\
& \sum_{i=1}^{M}\left\|\phi_{i}-\psi_{i}\right\|^{2}<\varepsilon,
\end{aligned}
$$

$\Psi$ is full spark.

Proof. Consider the collection of subsets of indices,

$$
\left\{\Lambda_{j} \subset[M]:\left|\Lambda_{j}\right|=\operatorname{dim} \operatorname{sym}\left(\mathbb{H}^{N \times N}\right)\right\} .
$$


For each $j,\left\{\phi_{i} \phi_{i}^{*}\right\}_{i \in \Lambda_{j}}$ is Riesz. Suppose that $\left\{\phi_{i} \phi_{i}^{*}\right\}_{i \in \Lambda_{j}}$ has lower Riesz bound $A_{j}$. Choose $\varepsilon<\min _{j} A_{j} / 2$ and a collection $\left\{\psi_{i}\right\}_{i=1}^{M}$ such that $\sum_{i=1}^{M}\left\|\phi_{i}-\psi_{i}\right\|^{2}<\varepsilon$. Then we have $\sum_{i \in \Lambda_{j}}\left\|\phi_{i}-\psi_{i}\right\|^{2}<\varepsilon$, and thus by Proposition 2.7.3, $\left\{\psi_{i} \psi_{i}^{*}\right\}_{i \in \Lambda_{j}}$ is Riesz for each $j$ as desired.

Combining Lemma 2.9.5 and Lemma 2.9.6, we get the following:

Theorem 2.9.7. The collection of frames $\left\{\phi_{i}\right\}_{i=1}^{M}$ with $M \geq \operatorname{dim} \operatorname{sym}\left(\mathbb{H}^{N \times N}\right)$ such that $\left\{\phi_{i} \phi_{i}^{*}\right\}_{i=1}^{M}$ is full spark, is open and dense in $\bigotimes_{i=1}^{M} S^{N-1}$. 


\section{Chapter 3}

\section{The Distributions of Hilbert Space Frame Vectors and Frame Coefficients}

The following work was developed jointly with Kevin Brewster, Peter Casazza and Lindsey Woodland, (see [4] for more). The most fundamental notion for a Hilbert space frame $\Phi=\left\{\phi_{i}\right\}_{i=1}^{M}$ for $\mathbb{H}^{N}$ is the sequence of frame coefficients for a vector $\mathrm{x}$, namely $\left\{\left\langle x, \phi_{i}\right\rangle\right\}_{i=1}^{M}$. But we know little about the distribution of these coefficients, even for very specific frames. In this chapter, we will make a detailed study of the distribution of the frame coefficients for general frames and then strengthen the results for several special classes of frames, including unit norm tight frames and equiangular frames. For this we use the concept of majorization. We will also study the distributions of products of frame coefficients for different vectors $x$ and $y$. We will then make a detailed study of the square sums of the distances from a vector $x$ to the frame vectors and discover that, generally speaking, these sums are nearly equal for all vectors. 


\subsection{Estimating the Number of Non-Zero Frame Coefficients}

In this section we will provide estimates on the number of indices for which the frame coefficients are non-zero. We will be looking for vectors producing the minimal number of non-zero frame coefficients because there is always a dense set of vectors that have non-zero inner products with all the frame vectors. For example, if $\left\{\phi_{i}\right\}_{i=1}^{M}$ is a frame for $\mathbb{H}^{N}$, then $\phi_{i}^{\perp}$ is a hyperplane for every $i \in[M]$. If we choose

$$
x \notin \cup_{i=1}^{M} \phi_{i}^{\perp},
$$

then

$$
\left|\left\{i \in[M]:\left\langle x, \phi_{i}\right\rangle \neq 0\right\}\right|=M .
$$

In general, given a vector $x$, there may be only a few indices for which $\left\langle x, \phi_{i}\right\rangle \neq 0$.

Example 3.1.1. Given $K$ copies of an orthonormal basis $\left\{e_{i}\right\}_{i=1}^{N}$ for $\mathbb{H}^{N}$, say $\left\{e_{i j}\right\}_{1 \leq i \leq N, 1 \leq j \leq K}$, and choosing $x=e_{1}$ gives

$$
\left\langle x, e_{i j}\right\rangle=0, \quad \forall i \in\{2, \ldots, N\}, j \in\{1, \ldots, K\} .
$$

That is, we have only $K$ non-zero coefficients out of a total of $K N$. In other words, there are $K N / N$ non-zero coefficients. Notice that this frame has lower (and upper) frame bound $K$. We will see that having $K$ non-zero coefficients is not a coincidence and, in fact, is minimal in this case.

Theorem 3.1.2. Let $\Phi=\left\{\phi_{i}\right\}_{i=1}^{M}$ be a frame in $\mathbb{H}^{N}$ with frame bounds $A, B$ and set $D:=\max \left\{\left\|\phi_{i}\right\|^{2}: i \in[M]\right\}$. For any unit norm $x \in \mathbb{H}^{N}$, define

$$
J_{x}:=\left\{i \in[M]:\left\langle x, \phi_{i}\right\rangle \neq 0\right\} .
$$


Then

$$
\left|J_{x}\right| \geq \frac{A}{D}
$$

Hence, if $\Phi$ is a unit norm frame, then $\left|J_{x}\right| \geq A$, and if it is a unit norm tight frame, then $\left|J_{x}\right| \geq M / N$.

Moreover, if we have equality in (3.1), then the sub-collection of frame vectors $\left\{\phi_{i}: i \in J_{x}\right\}$ spans a one-dimensional space.

Proof. Pick a unit norm $x \in \mathbb{H}^{N}$ and set $J_{x}:=\left\{1 \leq i \leq M:\left\langle x, \phi_{i}\right\rangle \neq 0\right\}$. Then

$$
\begin{aligned}
A & =A\|x\|^{2} \\
& \leq \sum_{i=1}^{M}\left|\left\langle x, \phi_{i}\right\rangle\right|^{2} \\
& =\sum_{i \in J_{x}}\left|\left\langle x, \phi_{i}\right\rangle\right|^{2} \\
& \leq \sum_{i \in J_{x}}\|x\|^{2}\left\|\phi_{i}\right\|^{2} \\
& =\sum_{i \in J_{x}}\left\|\phi_{i}\right\|^{2} \\
& \leq D\left|J_{x}\right| .
\end{aligned}
$$

Hence, $A / D$ is a lower bound for $\left|J_{x}\right|$ independent of each unit norm $x \in \mathbb{H}^{N}$. It follows that (3.1) holds true.

Concerning the "moreover part," if we have equality in (3.2), then

$$
\left|\left\langle x, \phi_{i}\right\rangle\right|^{2}=\left\|\phi_{i}\right\|^{2}, \text { for all } i \in J_{x}
$$

It follows that $\phi_{i}=c_{i} x$, for some $\left|c_{i}\right|=1$ and all $i \in J_{x}$.

Note that Example 3.1.1 satisfies the minimal number of non-zero frame coefficients in the theorem. We may be interested in not only knowing when coefficients are non- 
zero, but also when they are bounded away from zero. We provide lower bounds in the following theorem, which can be viewed as a generalization of the previous.

Theorem 3.1.3. Let $C \in(0,1)$ and let $\Phi=\left\{\phi_{i}\right\}_{i=1}^{M}$ be a frame for $\mathbb{H}^{N}$ with frame bounds $A, B$ and set $D:=\max \left\{\left\|\phi_{i}\right\|^{2}: i \in[M]\right\}$. For a unit norm $x \in \mathbb{H}^{N}$, define $K_{x}:=\left\{i \in[M]:\left|\left\langle x, \phi_{i}\right\rangle\right|^{2}>C A / M\right\}$. We have

$$
\left|K_{x}\right| \geq(1-C) \frac{A}{D}
$$

In particular, if $\Phi$ is a unit norm tight frame, then

$$
\left|K_{x}\right| \geq(1-C) \frac{M}{N}
$$

Proof. For unit norm $x \in \mathbb{H}^{N}$, we have

$$
\sum_{i \in K_{x}^{c}}\left|\left\langle x, \phi_{i}\right\rangle\right|^{2} \leq \frac{C A}{M}\left|K_{x}^{c}\right| \quad \text { and } \quad \sum_{i \in K_{x}}\left|\left\langle x, \phi_{i}\right\rangle\right|^{2} \leq D\left|K_{x}\right| .
$$

Hence,

$$
\begin{aligned}
A & =A\|x\|^{2} \\
& \leq \sum_{i=1}^{M}\left|\left\langle x, \phi_{i}\right\rangle\right|^{2} \\
& =\sum_{i \in K_{x}}\left|\left\langle x, \phi_{i}\right\rangle\right|^{2}+\sum_{i \in K_{x}^{c}}\left|\left\langle x, \phi_{i}\right\rangle\right|^{2} \\
& \leq D\left|K_{x}\right|+\frac{C A}{M}\left|K_{x}^{c}\right| \\
& =D\left|K_{x}\right|+\frac{C A}{M}\left(M-\left|K_{x}\right|\right) \\
& =\left(D-\frac{C A}{M}\right)\left|K_{x}\right|+C A .
\end{aligned}
$$

It follows that

$$
A(1-C) \leq\left(D-\frac{C A}{M}\right)\left|K_{x}\right| .
$$


By the definition of $K_{x}$ and equation (3.4), we may deduce that $D>C A / M$. Consequently,

$$
\left|K_{x}\right| \geq(1-C) \frac{A}{D-C A / M} \geq(1-C) \frac{A}{D} .
$$

Since the lower bound in (3.5) is independent of the unit norm $x \in \mathbb{H}^{N}$, the validity of (3.3) follows.

\subsection{The Distribution of the Frame Coefficients}

In this section we will classify the distribution of the frame coefficients using $m a-$ jorization. The theory of majorization is interesting in itself; however, we will need only a small portion of this theory. The interested reader should refer to [17] for a detailed study of majorization inequalities and their applications.

Definition 3.2.1. Let $a=\left(a_{i}\right)_{i=1}^{M}$ and $b=\left(b_{i}\right)_{i=1}^{M}$ be vectors with non-negative, nonincreasing components.

(i) We say a majorizes $b$ and write $a \succ b$ if

$$
\sum_{i=1}^{k} a_{i} \geq \sum_{i=1}^{k} b_{i}, \quad \forall k \in\{1,2, \ldots, M\} \quad \text { and } \quad \sum_{i=1}^{M} a_{i}=\sum_{i=1}^{M} b_{i} .
$$

(ii) We say $a$ weakly majorizes $b$ and write $a \succ_{W} b$ if

$$
\sum_{i=1}^{k} a_{i} \geq \sum_{i=1}^{k} b_{i}, \quad \forall k \in\{1,2, \ldots, M\}
$$

If the two vectors do not have the same number of elements, we agree to pad the shorter vector with zeros so that they have the same length. 
It will be important for our work to understand what happens when partial sums of the majorization vectors are equal.

Proposition 3.2.2. Let $a:=\left(a_{i}\right)_{i=1}^{M} \in \mathbb{R}^{M}$ be a non-negative, non-increasing sequence of numbers and let $b:=(A, A, \ldots, A) \in \mathbb{R}^{M}$, where $A>0$, and assume $a \succ_{W} b$. If there exists $m \in\{1, \ldots, M\}$ so that $\sum_{i=1}^{m} a_{i}=m A$, then $a_{i}=A$ for every $i \in\{m+1, \ldots, M\}$.

Proof. Assume there exists $m \in\{1, \ldots, M\}$ so that $\sum_{i=1}^{m} a_{i}=m A$. Then $a_{m} \leq A$. Hence, $a_{i} \leq A$ for every $i \in\{m+1, \ldots, M\}$. Assume that $a_{i}<A$ for some $i \in$ $\{m+1, \ldots, M\}$, then

$$
\begin{aligned}
M A & =\sum_{i=1}^{M} A \\
& \leq \sum_{i=1}^{M} a_{i} \\
& =\sum_{i=1}^{m} a_{i}+\sum_{i=m+1}^{M} a_{i} \\
& <m A+(M-m) A \\
& =M A,
\end{aligned}
$$

which is a contradiction. Thus, $a_{i}=A$ for every $i \in\{m+1, \ldots, M\}$.

The following proposition establishes a relationship between the largest eigenvalue of the frame operator and the number of frame coefficients of modulus one.

Proposition 3.2.3. Let $\left\{\phi_{i}\right\}_{i=1}^{M}$ be a unit norm frame in $\mathbb{H}^{N}$ whose frame operator has eigenvalues $\lambda_{1} \geq \cdots \geq \lambda_{N}$, and let $x \in \mathbb{H}^{N}$ be unit norm. Define the vector $a:=\left(a_{i}\right)_{i=1}^{M} \in \mathbb{R}^{M}$ to be $\left(\left|\left\langle x, \phi_{i}\right\rangle\right|^{2}\right)_{i=1}^{M}$, arranged in non-increasing order. Then $a_{i}=1$ for at most $\left\lfloor\lambda_{1}\right\rfloor$ indices $i$, where $\lfloor\cdot\rfloor$ represents the least integer function. 
Proof. By definition, $\sum_{i=1}^{M}\left|\left\langle x, \phi_{i}\right\rangle\right|^{2} \leq \lambda_{1}\|x\|^{2}=\lambda_{1}$. Suppose that $a_{i}=1$ for every $i \in\left\{1, \ldots,\left\lfloor\lambda_{1}\right\rfloor+1\right\}$, then

$$
\begin{aligned}
\lambda_{1} & \geq \sum_{i=1}^{M}\left|\left\langle x, \phi_{i}\right\rangle\right|^{2} \\
& =\sum_{i=1}^{\left\lfloor\lambda_{1}\right\rfloor+1}\left|\left\langle x, \phi_{i}\right\rangle\right|^{2}+\sum_{i=\left\lfloor\lambda_{1}\right\rfloor+2}^{M}\left|\left\langle x, \phi_{i}\right\rangle\right|^{2} \\
& =\left(\left\lfloor\lambda_{1}\right\rfloor+1\right)+\sum_{i=\left\lfloor\lambda_{1}\right\rfloor+2}^{M}\left|\left\langle x, \phi_{i}\right\rangle\right|^{2} \\
& >\lambda_{1},
\end{aligned}
$$

which is a contradiction.

We are ready to give one of the main majorization results for frame coefficients of unit norm vectors.

Theorem 3.2.4. Let $\left\{\phi_{i}\right\}_{i=1}^{M}$ be a frame for $\mathbb{H}^{N}$ with frame bounds $A \leq B$, and let

$$
b:=\left(\frac{A}{M}, \frac{A}{M}, \ldots, \frac{A}{M}\right) \in \mathbb{R}^{M} .
$$

For any unit norm $x \in \mathbb{H}^{N}$, define $a:=\left(a_{i}\right)_{i=1}^{M} \in \mathbb{R}^{M}$ to be $\left(\left|\left\langle x, \phi_{i}\right\rangle\right|^{2}\right)_{i=1}^{M}$, arranged in non-increasing order. Then $a \succ_{W} b$. In particular, $a_{1} \geq A / M$.

Moreover, if there exists an $m \in\{1, \ldots, M\}$ such that $\sum_{i=1}^{m} a_{i}=m(A / M)$, then $a_{i}=A / M$ for all $i \in\{m+1, \ldots, M\}$.

Proof. We proceed by way of contradiction. If there is an $m \in\{1, \ldots, M\}$ so that

$$
\sum_{i=1}^{m} a_{i}<\sum_{i=1}^{m} \frac{A}{M}=m \frac{A}{M}
$$

then $a_{m}<A / M$. Furthermore, it follows that

$$
a_{i} \leq a_{m}, \quad \forall i \in\{m, \ldots, M\}
$$


and we may write for any unit vector $x \in \mathbb{H}^{N}$

$$
\begin{aligned}
A & =A\|x\|^{2} \\
& \leq \sum_{i=1}^{M} a_{i} \\
& =\sum_{i=1}^{m} a_{i}+\sum_{i=m+1}^{M} a_{i} \\
& <m \frac{A}{M}+(M-m) a_{m} \\
& <m \frac{A}{M}+(M-m) \frac{A}{M} \\
& =A,
\end{aligned}
$$

which is a contradiction.

The "moreover" part follows from Proposition 3.2.2.

Corollary 3.2.5. Let $\left\{\phi_{i}\right\}_{i=1}^{M}$ be a unit norm tight frame in $\mathbb{H}^{N}$. For any unit norm $x \in \mathbb{H}^{N}$, if $a:=\left(a_{i}\right)_{i=1}^{M} \in \mathbb{R}^{M}$ is $\left(\left|\left\langle x, \phi_{i}\right\rangle\right|^{2}\right)_{i=1}^{M}$ arranged in non-increasing order and we set

$$
b:=\left(\frac{1}{N}, \frac{1}{N}, \ldots, \frac{1}{N}\right) \in \mathbb{R}^{M},
$$

then $a \succ_{W} b$.

Given a frame $\left\{\phi_{i}\right\}_{i=1}^{M}$ and a unit vector $x \in \mathbb{H}^{N}$, if $x$ is orthogonal to a number of frame vectors, we would expect the non-zero coefficients to start to grow, and they indeed do, as we shall see in the following proposition.

Proposition 3.2.6. Let $\left\{\phi_{i}\right\}_{i=1}^{M}$ be a frame for $\mathbb{H}^{N}$ with frame bounds $A, B$. For a given unit norm $x \in \mathbb{H}^{N}$, define $I:=\left\{i \in[M]: x \perp \phi_{i}\right\}$ and let $K=|I|$. Finally, let 
$a:=\left(a_{i}\right)_{i=1}^{M} \in \mathbb{R}^{M}$ be $\left(\left|\left\langle x, \phi_{i}\right\rangle\right|^{2}\right)_{i=1}^{M}$ arranged in non-increasing order and set

$$
b:=(\underbrace{\frac{A}{M-K}, \ldots, \frac{A}{M-K}}_{M-K \text { terms }}, \underbrace{0, \ldots, 0}_{K \text { terms }}) \in \mathbb{R}^{M} .
$$

Then $a \succ_{W} b$.

Proof. Without loss of generality, assume $I=\{M-K+1, \ldots, M\}$ (if not, apply a permutation to the indices $\{1, \ldots, M\})$. Thus, $I^{c}=\{1, \ldots, M-K\}$. Furthermore, since $\left(a_{i}\right)_{i=1}^{M}$ is in non-increasing order and $x \perp \phi_{i}$ for each $i \in I$, then $a_{i}=0$ for all $i \in I$. Being that $a_{i} \geq 0$ for all $i \in\{1, \ldots, M\}$, it suffices to show that

$$
\sum_{i=1}^{m} a_{i} \geq \sum_{i=1}^{m} \frac{A}{M-K}
$$

for all $m \in\{1, \ldots, M-K\}$. We proceed by way of contradiction. If there exists $m \in\{1, \ldots, M-K\}$ so that

$$
\sum_{i=1}^{m} a_{i}<\sum_{i=1}^{m} \frac{A}{M-K}=m \frac{A}{M-K}
$$

then $a_{m}<\frac{A}{M-K}$. Moreover, it follows that

$$
a_{i} \leq a_{m}, \quad \forall i \in\{m, \ldots, M-K\}
$$

and we may write for any unit vector $x \in \mathbb{H}^{N}$

$$
\begin{aligned}
A & =A\|x\|^{2} \\
& \leq \sum_{i=1}^{M} a_{i} \\
& =\sum_{i \in I^{c}} a_{i}+\sum_{i \in I} a_{i} \\
& =\sum_{i \in I^{c}} a_{i}
\end{aligned}
$$




$$
\begin{aligned}
& =\sum_{i=1}^{m} a_{i}+\sum_{i=m+1}^{M-K} a_{i} \\
& <\sum_{i=1}^{m} \frac{A}{M-K}+\sum_{i=m+1}^{M-K} a_{m} \\
& =m \frac{A}{M-K}+(M-K-m) a_{m} \\
& <m \frac{A}{M-K}+(M-K-m) \frac{A}{M-K} \\
& =A,
\end{aligned}
$$

which is a contradiction.

\subsection{Products of Frame Coefficients}

In this section, we will investigate the following: given a frame $\left\{\phi_{i}\right\}_{i=1}^{M}$ for $\mathbb{H}^{N}$, what can be said about the quantity

$$
\min _{\|x\|=1=\|y\|} \sum_{i=1}^{M}\left|\left\langle x, \phi_{i}\right\rangle\right|\left|\left\langle y, \phi_{i}\right\rangle\right| ?
$$

We start with a few basic observations.

Observation 3.3.1. If $M \leq 2 N-2$, we can always choose $x$ and $y$ such that $x \perp \phi_{i}$ for all $i \in I_{x} \subset\{1, \ldots, M\},\left|I_{x}\right| \leq N-1$, and $y \perp \phi_{i}$ for all $i \in I_{y} \subset\{1, \ldots, M\}$, $\left|I_{y}\right| \leq=N-1$, so that $I_{x} \cap I_{y}=\emptyset$ making the quantity (3.6) zero.

Observation 3.3.2. If we have a Hilbert space $\mathbb{H}^{N_{1}} \oplus \mathbb{H}^{N_{2}}$ and vectors $\left\{\phi_{i}\right\}_{i \in I}$ in $\mathbb{H}^{N_{1}}$ and $\left\{\phi_{i}\right\}_{i \in J}$ in $\mathbb{H}^{N_{2}}$, then by choosing $x \in \mathbb{H}^{N_{1}}$ and $y \in \mathbb{H}^{N_{2}}$ unit norm, we have

$$
\sum_{i \in I \cup J}\left|\left\langle x, \phi_{i}\right\rangle\right|\left|\left\langle y, \phi_{i}\right\rangle\right|=0
$$

In light of the above observations, nothing can be said about (3.6) in general. However, there are cases where we can produce meaningful results. We proceed 
analogously to the preceding sections in that we begin by estimating the number of non-zero summands. That is, we wish to know for how many $i \in[M]$ we have $\left|\left\langle x, \phi_{i}\right\rangle\right|\left|\left\langle y, \phi_{i}\right\rangle\right| \neq 0$

Definition 3.3.3. Let $\Phi=\left\{\phi_{i}\right\}_{i=1}^{M}$ be a frame for $\mathbb{H}^{N}$. We say that $\Phi$ has the complement property if for every $I \subset[M]$, with $|I|=N$ either $\left\{\phi_{i}\right\}_{i \in I}$ or $\left\{\phi_{i}\right\}_{i \in I^{c}}$ spans $\mathbb{H}^{N}$.

Proposition 3.3.4. Let $\left\{\phi_{i}\right\}_{i=1}^{M}$ be a frame for $\mathbb{H}^{N}$ with $M \geq 2 N-1$. The following are equivalent:

(i) $\left\{\phi_{i}\right\}_{i=1}^{M}$ has the complement property.

(ii) For every $x, y \in \mathbb{H}^{N}$, we have $\sum_{i=1}^{M}\left|\left\langle x, \phi_{i}\right\rangle\right|\left|\left\langle y, \phi_{i}\right\rangle\right| \neq 0$.

Moreover, if $\left\{\phi_{i}\right\}_{i=1}^{M}$ is full spark, then

$$
\left|\left\{i:\left|\left\langle x, \phi_{i}\right\rangle\right|\left|\left\langle y, \phi_{i}\right\rangle\right| \neq 0\right\}\right| \geq M-(2 N-2) .
$$

Proof.

(i) $\Rightarrow$ (ii): We will prove the contrapositive. Suppose there exist non-zero $x, y \in \mathbb{H}^{N}$ such that $\sum_{i=1}^{M}\left|\left\langle x, \phi_{i}\right\rangle\right|\left|\left\langle y, \phi_{i}\right\rangle\right|=0$, and define

$$
\begin{aligned}
& I_{x}:=\left\{1 \leq i \leq M:\left\langle x, \phi_{i}\right\rangle=0\right\}, \\
& I_{y}:=\left\{1 \leq i \leq M:\left\langle y, \phi_{i}\right\rangle=0\right\} .
\end{aligned}
$$

Case 1: If $I_{x} \cap I_{y}=\emptyset$, then $I_{y}=I_{x}^{c}$. Furthermore, $x \perp \phi_{i}$ for all $i \in I_{x}$ and, thus, $\operatorname{span}\left(\left\{\phi_{i}\right\}_{i \in I_{x}}\right) \neq \mathbb{H}^{N}$. Also, $y \perp \phi_{i}$ for all $i \in I_{y}=I_{x}^{c}$ and, thus, $\operatorname{span}\left(\left\{\phi_{i}\right\}_{i \in I_{x}^{c}}\right) \neq \mathbb{H}^{N}$. Therefore, there exists a partition $I_{x}, I_{x}^{c}$ of $\left\{\phi_{i}\right\}_{i=1}^{M}$, for which neither set spans $\mathbb{H}^{N}$. Hence, $\left\{\phi_{i}\right\}_{i=1}^{M}$ fails the complement property. 
Case 2: If $I_{x} \cap I_{y} \neq \emptyset$, then $\left(I_{y} \backslash\left(I_{x} \cap I_{y}\right)\right)=I_{x}^{c}$. Furthermore, $x \perp \phi_{i}$ for all $i \in I_{x}$ and, thus, $\operatorname{span}\left(\left\{\phi_{i}\right\}_{i \in I_{x}}\right) \neq \mathbb{H}^{N}$. Also, $y \perp \phi_{i}$ for all $i \in\left(I_{y} \backslash\left(I_{x} \cap I_{y}\right)\right)=I_{x}^{c}$ and, thus, $\operatorname{span}\left(\left\{\phi_{i}\right\}_{i \in I_{x}^{c}}\right) \neq \mathbb{H}^{N}$. Therefore, there exists a partition $I_{x}, I_{x}^{c}$ of $\left\{\phi_{i}\right\}_{i=1}^{M}$ for which neither set spans $\mathbb{H}^{N}$. Thus, $\left\{\phi_{i}\right\}_{i=1}^{M}$ fails the complement property.

(ii) $\Longrightarrow$ (i): Again by contrapositive. Suppose $\left\{\phi_{i}\right\}_{i=1}^{M}$ fails the complement property. This implies that there exists a partition $I, I^{c}$ of $\{1, \ldots, M\}$ such that $\operatorname{span}\left(\left\{\phi_{i}\right\}_{i \in I}\right) \neq$ $\mathbb{H}^{N}$ and $\operatorname{span}\left(\left\{\phi_{i}\right\}_{i \in I^{c}}\right) \neq \mathbb{H}^{N}$. Thus, there exist $x, y \in \mathbb{H}^{N}$ with the property that $x \perp\left\{\phi_{i}\right\}_{i \in I}$ and $y \perp\left\{\phi_{i}\right\}_{i \in I^{c}}$. Therefore, $\sum_{i=1}^{M}\left|\left\langle x, \phi_{i}\right\rangle\right|\left|\left\langle y, \phi_{i}\right\rangle\right|=0$, as desired.

For the "moreover" part, by full spark we have

$$
\left|\left\{i:\left|\left\langle x, \phi_{i}\right\rangle\right|=0\right\}\right| \leq N \quad \text { and } \quad\left|\left\{i:\left|\left\langle y, \phi_{i}\right\rangle\right|=0\right\}\right| \leq N,
$$

as desired.

We digress for a moment to point out an interesting connection to the problem of phaseless signal reconstruction. Introduced in [2], Balan, Casazza and Ediden proved necessary and sufficient conditions for a frame to do phaseless reconstruction. That is, if we define the map

$$
A: \mathbb{R}^{N} /\{ \pm 1\} \rightarrow \mathbb{R}_{\geq 0}^{M}
$$

by

$$
A: x \mapsto\left(\left|\left\langle x, \phi_{i}\right\rangle\right|^{2}\right)
$$

then under what circumstances is this map injective? The authors demonstrate that $A$ is injective if and only if for every subset $I \subset[M]$, we have $\left\{\phi_{i}\right\}_{i \in I}$ or $\left\{\phi_{i}\right\}_{i \in I^{c}}$ spans. This is equivalent to (ii) in Proposition 3.3.4.

Next, we give an upper bound on the sum in equation (3.6). 
Lemma 3.3.5. Let $\left\{\phi_{i}\right\}_{i=1}^{M}$ be a frame in $\mathbb{H}^{N}$ with frame bounds $A, B$. Then

$$
\sup \left\{\sum_{i=1}^{M}\left|\left\langle x, \phi_{i}\right\rangle\left\|\left\langle y, \phi_{i}\right\rangle \mid: x, y \in \mathbb{H}^{N},\right\| x\|=1=\| y \|\right\} \leq B .\right.
$$

Proof. Let $x, y \in \mathbb{H}^{N}$ be unit norm. Invoking Hölder's inequality gives

$$
\begin{aligned}
\sum_{i=1}^{M}\left|\left\langle x, \phi_{i}\right\rangle\right|\left|\left\langle y, \phi_{i}\right\rangle\right| & \leq\left(\sum_{i=1}^{M}\left|\left\langle x, \phi_{i}\right\rangle\right|^{2}\right)^{1 / 2}\left(\sum_{i=1}^{M}\left|\left\langle y, \phi_{i}\right\rangle\right|^{2}\right)^{1 / 2} \\
& \leq B^{1 / 2} B^{1 / 2}=B
\end{aligned}
$$

The following proposition concerns another upper bound estimate for the summation in (3.6). It should be noted that the proposition is neither a stronger nor a weaker version of Lemma 3.3.5. This can be seen by making particular choices of $M, N$ that make the bounds in the lemma better than the bounds in the proposition, and vice versa.

Proposition 3.3.6. Let $M \geq 2 N-1$, and let $\left\{\phi_{i}\right\}_{i=1}^{M}$ be a frame for $\mathbb{H}^{N}$ with frame bounds $A, B$. Then

$$
\sup _{\|x\|=1=\|y\|}\left\{\sum_{i=1}^{M}\left|\left\langle x, \phi_{i}\right\rangle\right|\left|\left\langle y, \phi_{i}\right\rangle\right|\right\} \leq(M-2 N+2) \sqrt{\frac{B}{N}} .
$$

Proof. Pick unit vectors $x, y \in \mathbb{H}^{N}$ so that $x \perp \phi_{i}$ for all $i \in\{1, \ldots, N-1\}$ and $y \perp \phi_{i}$ for all $i \in\{M-N+2, \ldots, M\}$. It follows that

$$
B=B\|x\|^{2} \geq \sum_{i=1}^{M}\left|\left\langle x, \phi_{i}\right\rangle\right|^{2}=\sum_{i=N}^{M}\left|\left\langle x, \phi_{i}\right\rangle\right|^{2} .
$$

Suppose that $\left|\left\langle x, \phi_{i}\right\rangle\right|^{2}>\frac{B}{N}$ for all $i \in\{N, \ldots, M\}$. Then

$$
B \geq \sum_{i=N}^{M}\left|\left\langle x, \phi_{i}\right\rangle\right|^{2}>(M-N+1) \frac{B}{N} \geq N \frac{B}{N}=B
$$


which is a contradiction. Hence, there exists $i_{1} \in\{N, \ldots, M\}$ such that $\left|\left\langle x, \phi_{i_{1}}\right\rangle\right|^{2} \leq$ $\frac{B}{N}$. If the third inequality in (3.7) is strict, then using a similar proof by contradiction (as in (3.7)) at most $M-2 N+1$ more times, we obtain that there exist indices $i_{2}, \ldots, i_{M-2 N+2}$ all not equal to $i_{1}$ so that $\left|\left\langle x, \phi_{i_{j}}\right\rangle\right|^{2} \leq \frac{B}{N}$ for every $j \in\{1, \ldots, M-$ $2 N+2\}$. Without loss of generality, we may assume $i_{1}=N, i_{2}=N+1, \ldots, i_{M-2 N+2}=$ $M-N+1$. Then

$$
\begin{aligned}
\sum_{i=1}^{M}\left|\left\langle x, \phi_{i}\right\rangle\right|\left|\left\langle y, \phi_{i}\right\rangle\right| & =\sum_{i=N}^{M-N+1}\left|\left\langle x, \phi_{i}\right\rangle\right|\left|\left\langle y, \phi_{i}\right\rangle\right| \\
& \leq \sum_{i=N}^{M-N+1}\left|\left\langle x, \phi_{i}\right\rangle\right| \\
& \leq(M-2 N+2) \sqrt{\frac{B}{N}}
\end{aligned}
$$

Taking the supremum over all $x, y \in \mathbb{H}^{N}$ so that $\|x\|=1=\|y\|$ in (3.8) gives the desired result.

Remark 3.3.7. Now we compare the bounds in Lemma 3.3.5 with Proposition 3.3.6. Let $M \geq 2 N-1$, and let $\left\{\phi_{i}\right\}_{i=1}^{M}$ be a unit norm tight frame for $\mathbb{H}^{N}$. If $M-\sqrt{M}+2 \geq$ $2 N$, then $(M-2 N+2) \frac{\sqrt{M}}{N} \geq \frac{M}{N}$. Hence, $\frac{M}{N}$ is a better upper bound for

$$
\sup _{x, y \in \mathbb{H}^{N},\|x\|=1=\|y\|}\left\{\sum_{i=1}^{M}\left|\left\langle x, \phi_{i}\right\rangle\right|\left|\left\langle y, \phi_{i}\right\rangle\right|\right\} .
$$

Moreover, when $M-\sqrt{M}+2<2 N$ then $(M-2 N+2) \frac{\sqrt{M}}{N}<\frac{N}{M}$. Hence, $(M-2 N+$ 2) $\frac{\sqrt{M}}{N}$ is a better bound for this supremum.

We now consider lower bounds for the summation in (3.6) in the case of tight frames. The next proposition demonstrates that this quantity is at least the product of the tight frame bound and the inner product of the unit vectors. 
Proposition 3.3.8. If $\left\{\phi_{i}\right\}_{i=1}^{M}$ is a unit norm tight frame in $\mathbb{H}^{N}$, then for any unit norm $x, y \in \mathbb{H}^{N}$, we have

$$
\frac{M}{N}|\langle x, y\rangle| \leq \sum_{i=1}^{M}\left|\left\langle x, \phi_{i}\right\rangle\right|\left|\left\langle y, \phi_{i}\right\rangle\right|
$$

Proof. Let $x, y \in \mathbb{H}^{N}$ be unit norm and consider

$$
\begin{aligned}
\frac{M}{N}|\langle x, y\rangle| & =\left|\left\langle x, \frac{M}{N} y\right\rangle\right| \\
& =\left|\left\langle x, \sum_{i=1}^{M}\left\langle y, \phi_{i}\right\rangle \phi_{i}\right\rangle\right| \\
& =\left|\sum_{i=1}^{M}\left\langle x, \phi_{i}\right\rangle \overline{\left\langle y, \phi_{i}\right\rangle}\right| \\
& \leq \sum_{i=1}^{M}\left|\left\langle x, \phi_{i}\right\rangle\right|\left|\left\langle y, \phi_{i}\right\rangle\right| .
\end{aligned}
$$

Continuing our investigation for lower bounds in the summation in equation (3.6), we make a slight modification in the following lemma. We will consider that $y$ is a fixed element of our frame and allow $x$ to vary. Recall that if $\left\{\phi_{i}\right\}_{i=1}^{M}$ is an equiangular tight frame for $\mathbb{H}^{N}$, then for $i \neq j$ we have

$$
\left|\left\langle\phi_{i}, \phi_{j}\right\rangle\right|^{2}=\frac{M-N}{N(M-1)}
$$

Lemma 3.3.9. Let $\left\{\phi_{i}\right\}_{i=1}^{M}$ be an equiangular tight frame in $\mathbb{H}^{N}$, and fix $j \in\{1, \ldots, M\}$. Then

$$
\inf \left\{\sum_{i=1}^{M}\left|\left\langle\phi_{j}, \phi_{i}\right\rangle\right|\left|\left\langle x, \phi_{i}\right\rangle\right|: x \in \mathbb{H}^{N},\|x\|=1\right\} \geq \frac{M}{N} \sqrt{\frac{M-N}{N(M-1)}} .
$$

In particular, when

(i) $M=2 N$, we get

$$
\inf \left\{\sum_{i=1}^{M}\left|\left\langle\phi_{j}, \phi_{i}\right\rangle\right|\left|\left\langle x, \phi_{i}\right\rangle\right|: x \in \mathbb{H}^{N},\|x\|=1\right\} \geq \frac{2}{\sqrt{2 N-1}} ;
$$


(ii) $M=\frac{N(N+1)}{2}$, we get

$$
\inf \left\{\sum_{i=1}^{M}\left|\left\langle\phi_{j}, \phi_{i}\right\rangle\right|\left|\left\langle x, \phi_{i}\right\rangle\right|: x \in \mathbb{H}^{N},\|x\|=1\right\} \geq \frac{N+1}{2 \sqrt{N+2}} .
$$

Proof. Fix a unit norm $x \in \mathbb{H}^{N}$ and fix $j \in\{1, \ldots, M\}$. Next, define $I:=\{1, \ldots, M\} \backslash$ $\{j\}$ and $c:=\sqrt{\frac{M-N}{N(M-1)}}$. Consider

$$
\begin{aligned}
\sum_{i=1}^{M}\left|\left\langle\phi_{j}, \phi_{i}\right\rangle\right|\left|\left\langle x, \phi_{i}\right\rangle\right| & =\sum_{i \in I}\left|\left\langle\phi_{j}, \phi_{i}\right\rangle\right|\left|\left\langle x, \phi_{i}\right\rangle\right|+\left|\left\langle\phi_{j}, \phi_{j}\right\rangle\right|\left|\left\langle x, \phi_{j}\right\rangle\right| \\
& =c \sum_{i \in I}\left|\left\langle x, \phi_{i}\right\rangle\right|+\left|\left\langle x, \phi_{j}\right\rangle\right| \\
& =c \sum_{i=1}^{M}\left|\left\langle x, \phi_{i}\right\rangle\right|+(1-c)\left|\left\langle x, \phi_{j}\right\rangle\right| \\
& \geq c \sum_{i=1}^{M}\left|\left\langle x, \phi_{i}\right\rangle\right| \geq c \sum_{i=1}^{M}\left|\left\langle x, \phi_{i}\right\rangle\right|^{2} \\
& =c \frac{M}{N} .
\end{aligned}
$$

This is a lower bound for the set $\left\{\sum_{i=1}^{M}\left|\left\langle\phi_{j}, \phi_{i}\right\rangle\right|\left|\left\langle x, \phi_{i}\right\rangle\right|: x \in \mathbb{H}^{N},\|x\|=1\right\}$, which is independent of the unit norm $x \in \mathbb{H}^{N}$.

\subsection{Distance Between Vectors and Frame Vectors}

In this section, we will give estimates of the squared sums of the distances between a vector and the frame vectors. We wil discover some surprising uniformities for the equiangular case.

The following lemma establishes a relationship between the coefficients of a collection of vectors and the norms and inner products of these vectors. While interesting in its own right, this lemma serves as a tool in Proposition 3.4.2.

Lemma 3.4.1. Let $\left\{\phi_{i}\right\}_{i=1}^{M}$ be a collection of vectors in $\mathbb{R}^{N}$ whose components are 
given as $\phi_{i}=\left(\phi_{i}(1), \ldots, \phi_{i}(N)\right)$. Then

$$
\begin{aligned}
\sum_{j=1}^{N}\left(\sum_{i=1}^{M} \phi_{i}(j)\right)^{2} & =\sum_{i=1}^{M} \sum_{j=1}^{N} \phi_{i}(j)\left(\sum_{k=1}^{M} \phi_{k}(j)\right) \\
& =\sum_{i=1}^{M}\left\|\phi_{i}\right\|^{2}+2 \sum_{1 \leq i<k \leq M}\left\langle\phi_{i}, \phi_{k}\right\rangle .
\end{aligned}
$$

Proof. Concerning the first equality, note that

$$
\begin{aligned}
\sum_{i=1}^{M} \sum_{j=1}^{N} \phi_{i}(j)\left(\sum_{k=1}^{M} \phi_{k}(j)\right) & =\sum_{j=1}^{N} \sum_{i=1}^{M} \phi_{i}(j)\left(\sum_{k=1}^{M} \phi_{k}(j)\right) \\
& =\sum_{j=1}^{N}\left(\sum_{k=1}^{M} \phi_{k}(j)\right) \sum_{i=1}^{M} \phi_{i}(j) \\
& =\sum_{j=1}^{N}\left(\sum_{i=1}^{M} \phi_{i}(j)\right)^{2} .
\end{aligned}
$$

For the last equality in (3.11), consider

$$
\begin{aligned}
\sum_{j=1}^{N}\left(\sum_{i=1}^{M} \phi_{i}(j)\right)^{2} & =\sum_{j=1}^{N}\left(\sum_{i=1}^{M} \phi_{i}(j)^{2}+2 \sum_{1 \leq i<k \leq M} \phi_{i}(j) \phi_{k}(j)\right) \\
& =\sum_{i=1}^{M} \sum_{j=1}^{N} \phi_{i}(j)^{2}+2 \sum_{1 \leq i<k \leq M} \sum_{j=1}^{N} \phi_{i}(j) \phi_{k}(j) \\
& =\sum_{i=1}^{M}\left\|\phi_{i}\right\|^{2}+2 \sum_{1 \leq i<k \leq M}\left\langle\phi_{i}, \phi_{k}\right\rangle,
\end{aligned}
$$

as desired.

The proposition below establishes upper and lower bounds for the sum of the squares of the distances between any unit vector and the frame vectors in an equiangular tight frame in terms of the ambient dimension and the modulus of the inner product between vectors.

Proposition 3.4.2. Let $\left\{\phi_{i}\right\}_{i=1}^{M}$ be an equiangular tight frame in $\mathbb{R}^{N}$. Then for any unit norm $x \in \mathbb{R}^{N}$, we have

$$
2(M-\sqrt{M[1+(M-1) c]}) \leq \sum_{i=1}^{M}\left\|x-\phi_{i}\right\|^{2} \leq 2(M+\sqrt{M[1+(M-1) c]}),
$$


where $c:=\sqrt{\frac{M-N}{N(M-1)}}=\left|\left\langle\phi_{i}, \phi_{j}\right\rangle\right|$, for all $i \neq j$.

Proof. Fix a unit norm $x \in \mathbb{R}^{N}$, and write the vectors $x, \phi_{1}, \ldots, \phi_{M} \in \mathbb{R}^{N}$ in terms of their components; that is, write $x=(x(1), \ldots, x(N))$ and $\phi_{i}=\left(\phi_{i}(1), \ldots, \phi_{i}(N)\right)$ for each $i$.

Next, define the functions $f, g: \mathbb{R}^{N} \longrightarrow \mathbb{R}$ by

$$
f(y):=\sum_{i=1}^{M}\left\|y-\phi_{i}\right\|^{2} \quad \text { and } \quad g(y):=\|y\|^{2} .
$$

Substituting our unit norm $x \in \mathbb{R}^{N}$ into the functions $f, g$ gives

$$
\begin{gathered}
f(x)=\sum_{i=1}^{M}\left(\|x\|^{2}+\left\|\phi_{i}\right\|^{2}-2\left\langle x, \phi_{i}\right\rangle\right)=2 M-2 \sum_{i=1}^{M} \sum_{j=1}^{N} x_{j} \phi_{i}(j) ; \\
g(x)=\|x\|^{2}=\sum_{i=1}^{N} x(i)^{2}=1 .
\end{gathered}
$$

At this stage, the main idea is to use the method of Lagrange multipliers on the function $f(x)$ subject to the constraint function $g(x)$ to identify any absolute extrema. To this end, we calculate $(\nabla f)(x)$ and $(\nabla g)(x)$ as

$$
\begin{gathered}
(\nabla f)(x)=-2\left(\sum_{i=1}^{M} \phi_{i}(1), \sum_{i=1}^{M} \phi_{i}(2), \ldots, \sum_{i=1}^{M} \phi_{i}(N)\right), \\
(\nabla g)(x)=2(x(1), x(2), \ldots, x(N)) .
\end{gathered}
$$

We solve the system of equations $(\nabla f)(x)=\lambda[(\nabla g)(x)], g(x)=1$, where $\lambda \in \mathbb{R}$, to obtain

$$
x(1)=-\frac{1}{\lambda} \sum_{i=1}^{M} \phi_{i}(1), \quad x(2)=-\frac{1}{\lambda} \sum_{i=1}^{M} \phi_{i}(2), \quad \ldots, \quad x(N)=-\frac{1}{\lambda} \sum_{i=1}^{M} \phi_{i}(N) .
$$

Substituting these coordinates into the function $g(x)=1$ gives

$$
1=\frac{1}{\lambda^{2}} \sum_{j=1}^{N}\left(\sum_{i=1}^{M} \phi_{i}(j)\right)^{2}
$$

By Lemma 3.4.1, we may write the above equation as

$$
\lambda^{2}=\sum_{i=1}^{M}\left\|\phi_{i}\right\|^{2}+2 \sum_{1 \leq i<k \leq M}\left\langle\phi_{i}, \phi_{k}\right\rangle=M+2 \sum_{1 \leq i<k \leq M}\left\langle\phi_{i}, \phi_{k}\right\rangle
$$


which implies that

$$
\lambda= \pm\left(M+2 \sum_{1 \leq i<k \leq M}\left\langle\phi_{i}, \phi_{k}\right\rangle\right)^{1 / 2} .
$$

Substituting the coordinates in (3.13) into the function $f$ given in (3.12) and using Lemma 3.4.1 yields

$$
\begin{aligned}
f(x) & =2 M-2 \sum_{i=1}^{M} \sum_{j=1}^{N}\left(-\frac{1}{\lambda} \sum_{k=1}^{M} \phi_{k}(j)\right) \phi_{i}(j) \\
& =2 M+\frac{2}{\lambda}\left[\sum_{i=1}^{M} \sum_{j=1}^{N} \phi_{i}(j)\left(\sum_{k=1}^{M} \phi_{k}(j)\right)\right] \\
& =2 M+\frac{2}{\lambda}\left[\sum_{i=1}^{M}\left\|\phi_{i}\right\|^{2}+2 \sum_{1 \leq i<k \leq M}\left\langle\phi_{i}, \phi_{k}\right\rangle\right] \\
& =2 M+\frac{2}{\lambda}\left[\lambda^{2}\right]=2(M+\lambda) .
\end{aligned}
$$

Thus, the global extrema for the function $f$ are $2(M+\lambda)$. Because $\lambda$ depends on $\left\langle\phi_{i}, \phi_{k}\right\rangle$ (and not on $\left|\left\langle\phi_{i}, \phi_{k}\right\rangle\right|$ ) for each $i \neq k, \lambda$ reaches its extrema when $\left\langle\phi_{i}, \phi_{k}\right\rangle=c$, where $c>0$. Because $\left\{\phi_{i}\right\}_{i=1}^{M}$ is an equiangular tight frame, $c=\sqrt{\frac{M-N}{N(M-1)}}$. Under these assumptions,

$$
\lambda= \pm \sqrt{M+2 \frac{M(M-1)}{2} c}= \pm \sqrt{M[1+(M-1) c]} .
$$

With $\lambda$ as such, the result follows.

Remark 3.4.3. In Proposition 3.4.2, the lower bound is always positive. Indeed, 2( $M-$ $\sqrt{M[1+(M-1) c]})>0$ if and only if $c<1$, which is satisfied a priori.

We establish slightly weaker bounds for Proposition 3.4.2 in the following corollary. Although these bounds are weaker, they are more accessible. 
Corollary 3.4.4. Let $\left\{\phi_{i}\right\}_{i=1}^{M}$ be an equiangular tight frame in $\mathbb{R}^{N}$. Then for any unit norm $x \in \mathbb{R}^{N}$, we have

$$
\begin{aligned}
2 M(1-\sqrt{2 c}) & <2(M-\sqrt{M[1+(M-1) c]}) \\
& \leq \sum_{i=1}^{M}\left\|x-\phi_{i}\right\|^{2} \\
& \leq 2(M+\sqrt{M[1+(M-1) c]}) \\
& <2 M(1+\sqrt{2 c}),
\end{aligned}
$$

where $c:=\sqrt{\frac{M-N}{N(M-1)}}\left(\right.$ i.e., $c=\left|\left\langle\phi_{i}, \phi_{j}\right\rangle\right|, \forall i, j \in\{1, \ldots, M\}$ such that $i \neq j$ ).

Proof. First note that

$$
c=\sqrt{\frac{M-N}{N(M-1)}} \geq \sqrt{\frac{1}{N(M-1)}} \geq \sqrt{\frac{1}{M(M-1)}}>\frac{1}{M} .
$$

For the right-hand side of the inequality in (3.14), we have

$$
\begin{aligned}
2(M+\sqrt{M[1+(M-1) c]}) & =2\left(M+M \sqrt{\frac{1}{M}+\frac{M-1}{M} c}\right) \\
& <2\left(M+M \sqrt{\frac{1}{M}+c}\right) \\
& <2(M+M \sqrt{2 c}) \\
& =2 M(1+\sqrt{2 c}) .
\end{aligned}
$$

For the left-hand side of the inequality in (3.14), we have

$$
\begin{aligned}
2(M-\sqrt{M[1+(M-1) c]}) & =2\left(M-M \sqrt{\frac{1}{M}+\frac{M-1}{M} c}\right) \\
& >2\left(M-M \sqrt{\frac{1}{M}+c}\right) \\
& >2(M-M \sqrt{2 c}) \\
& =2 M(1-\sqrt{2 c}),
\end{aligned}
$$


which is as desired. The last order of business is to check that $2 M(1-\sqrt{2 c})>0$, which happens if and only if $c<1 / 2$. We consider the following scenarios:

Case 1: $N \geq 4$. We proceed by way of contradiction. Note that $c \geq 1 / 2$ if and only if $M \geq \frac{M N}{4}+\frac{3 N}{4}$. If $N \geq 4$ and $c \geq 1 / 2$, then

$$
M \geq \frac{M N}{4}+\frac{3 N}{4} \geq M+3
$$

which is a contradiction. Therefore, $c<1 / 2$ when $N \geq 4$ (which forces $M \geq 4$ ).

Case 2: $N=3$. Note that $c<1 / 2$ if and only if $4 M-3 N-N M<0$. Thus, for $N=3$, we have $4 M-3(3)-3 M<0$ if and only if $M<9$. Recall in $\mathbb{R}^{3}$, that it is known that the maximum number of vectors in an equiangular unit norm tight frame is 6 , (see [18]). Hence, $c<1 / 2$ when $N=3$ (hence, $3 \leq M \leq 6<9$ ).

Case 3: $N=2$. Recall that in $\mathbb{R}^{2}$, it is known that the maximum number of vectors in an equiangular unit norm tight frame is 3 (see [18]). Also, from Case 2, we know that $c<1 / 2$ if and only if $4 M-3 N-N M<0$. For $N=2$, this becomes $4 M-3(2)-2 M<$ 0 , which happens if and only if $M<3$. Consequently, $c<1 / 2$ when $N=2$ and $M=2$.

This finishes the proof of Corollary 3.4.4.

Remark 3.4.5. For the special case $N=2$ and $M=3$, it follows that $c=1 / 2$ (see $(3.9))$ and

$$
\frac{1}{M}+c=\frac{1}{3}+\frac{1}{2}=\frac{5}{6}=\frac{5}{3} \cdot \frac{1}{2}=\frac{5}{3} c
$$

Thus, we actually get improved bounds when $N=2$ and $M=3$; specifically, see 
(3.15) and (3.16).

$$
\begin{aligned}
2 M\left(1-\sqrt{\frac{5}{3} c}\right) & \leq 2(M-\sqrt{M[1+(M-1) c]}) \\
& \leq \sum_{i=1}^{M}\left\|x-\phi_{i}\right\|^{2} \\
& \leq 2(M+\sqrt{M[1+(M-1) c]}) \\
& \leq 2 M\left(1+\sqrt{\frac{5}{3} c}\right) .
\end{aligned}
$$

The next corollary gives a surprising identity exhibited by simplex frames. Recall that up to multiplication by a unitary operator and switching (replacing a vector by its additive inverse) there is only one unit norm tight frame with $N+1$ elements in $\mathbb{R}^{N}$ (see [8]). This frame can be obtained in the following way: let $\left\{e_{i}\right\}_{i=1}^{N+1}$ be the standard orthonormal basis for $\mathbb{R}^{N+1}$, and let $P$ be the rank-one orthogonal projection onto $\operatorname{span}\left(\sum_{i=1}^{N+1} e_{i}\right)$. Then the vectors

$$
\left\{\phi_{i}\right\}_{i=1}^{N+1}:=\left\{\frac{(I-P) e_{i}}{\left\|(I-P) e_{i}\right\|}\right\}_{i=1}^{N+1}
$$

form an equiangular tight frame for $\mathbb{R}^{N}$. This frame is commonly referred to as the simplex frame in $\mathbb{R}^{N}$.

Corollary 3.4.6. Let $\left\{\phi_{i}\right\}_{i=1}^{N+1}$ be the simplex frame in $\mathbb{R}^{N}$. Then for any unit norm $x \in \mathbb{R}^{N}$, we have

$$
\sum_{i=1}^{N+1}\left\|x-\phi_{i}\right\|^{2}=2(N+1) .
$$

Proof. This follows immediately from Proposition 3.4.2 and the fact that $c=-\frac{1}{N}=-\frac{1}{M-1}$ for the simplex in $\mathbb{R}^{N}$.

There is a significant generalization of the above corollary for the case where the frame vectors sum to zero. 
Theorem 3.4.7. Let $\left\{\phi_{i}\right\}_{i=1}^{M}$ be a unit norm tight frame in $\mathbb{H}^{N}$, and assume $\sum_{i=1}^{M} \phi_{i}=$ 0. Then $\sum_{i=1}^{M}\left\|x-\phi_{i}\right\|^{2}=2 M$ for any unit norm $x \in \mathbb{H}^{N}$.

Proof. For any unit norm $x \in \mathbb{H}^{N}$, we have

$$
\begin{aligned}
\sum_{i=1}^{M}\left\|x-\phi_{i}\right\|^{2} & =\sum_{i=1}^{M}\|x\|^{2}+\left\|\phi_{i}\right\|^{2}-\left\langle x, \phi_{i}\right\rangle-\left\langle\phi_{i}, x\right\rangle \\
& =\sum_{i=1}^{M}\left(2-2 \operatorname{Re}\left(\left\langle x, \phi_{i}\right\rangle\right)\right) \\
& =2 M-2 \operatorname{Re}\left\langle x, \sum_{i=1}^{M} \phi_{i}\right\rangle \\
& =2 M-2 \operatorname{Re}\langle x, 0\rangle=2 M
\end{aligned}
$$

Finally, in this setting, we can get very accurate approximations for the sums of squared products of distances from vectors to the frame vectors.

Theorem 3.4.8. Let $\left\{\phi_{i}\right\}_{i=1}^{M}$ be a unit norm tight frame in $\mathbb{R}^{N}$, and assume that $\sum_{i=1}^{M} \phi_{i}=0$. Then for any unit norm $x \in \mathbb{H}^{N}$,

$$
4 M\left(1-\frac{1}{N}\right) \leq \sum_{i=1}^{M}\left\|x-\phi_{i}\right\|^{2}\left\|y-\phi_{i}\right\|^{2} \leq 4 M\left(1+\frac{1}{N}\right)
$$

Proof. First, note that for each $i \in\{1, \ldots, M\}$ and each $x \in \mathbb{R}^{N}$ unit norm, we have

$$
\left\|x-\phi_{i}\right\|^{2}=\|x\|^{2}+\left\|\phi_{i}\right\|^{2}-2\left\langle x, \phi_{i}\right\rangle=2\left(1-\left\langle x, \phi_{i}\right\rangle\right)
$$

Using this, we may write

$$
\begin{aligned}
\sum_{i=1}^{M}\left\|x-\phi_{i}\right\|^{2}\left\|y-\phi_{i}\right\|^{2} & =\sum_{i=1}^{M} 2\left(1-\left\langle x, \phi_{i}\right\rangle\right) 2\left(1-\left\langle y, \phi_{i}\right\rangle\right) \\
& =4 \sum_{i=1}^{M}\left(1-\left\langle x, \phi_{i}\right\rangle-\left\langle y, \phi_{i}\right\rangle+\left\langle x, \phi_{i}\right\rangle\left\langle y, \phi_{i}\right\rangle\right)
\end{aligned}
$$




$$
\begin{aligned}
& =4\left(M-0-0+\left\langle x, \sum_{i=1}^{M}\left\langle y, \phi_{i}\right\rangle \phi_{i}\right\rangle\right) \\
& =4\left(M+\left\langle x, \frac{M}{N} y\right\rangle\right) \\
& =4 M\left(1+\frac{1}{N}\langle x, y\rangle\right) .
\end{aligned}
$$

Using Cauchy-Schwarz and the fact that $x, y \in \mathbb{R}^{N}$ are unit norm, we may deduce that $-1 \leq\langle x, y\rangle \leq 1$. Combining this with the above calculation gives the desired result. 


\section{Bibliography}

[1] Boris Alexeev, Jameson Cahill, and Dustin G Mixon. Full spark frames. Journal of Fourier Analysis and Applications, 18(6):1167-1194, 2012.

[2] Radu Balan, Pete Casazza, and Dan Edidin. On signal reconstruction without phase. Applied and Computational Harmonic Analysis, 20(3):345-356, 2006.

[3] Alexander Barg, Kasso Okoudjou, and Wei-Hsuan Yu. Finite two-distance tight frames. arXiv preprint arXiv:1402.3521, 2014.

[4] Kevin Brewster, Peter G. Casazza, Eric Pinkham, and Lindsey Woodland. The distributions of hilbert space frame vectors and frame coefficients.

[5] Jameson Cahill. Frames and projections. ProQuest LLC, Ann Arbor, MI, 2013. Thesis (Ph.D.)-University of Missouri - Columbia.

[6] Jameson Cahill and Xuemei Chen. A note on scalable frames. arXiv preprint arXiv:1301.7292, 2013.

[7] Peter G. Casazza, Matthew Fickus, Jelena Kovačević, Manuel T. Leon, and Janet C. Tremain. A physical interpretation of tight frames. In Harmonic Analysis and Applications, Appl. Numer. Harmon. Anal., pages 51-76. Birkhäuser Boston, Boston, MA, 2006. 
[8] Peter G Casazza and Jelena Kovacevic. Uniform tight frames with erasures. preprint, 2001.

[9] Peter G. Casazza and Nicole Leonhard. Classes of finite equal norm Parseval frames. In Frames and Operator Theory in Analysis and Signal Processing, volume 451 of Contemp. Math., pages 11-31. Amer. Math. Soc., Providence, RI, 2008.

[10] Peter G Casazza, Eric Pinkham, and Brian Tuomanen. Riesz outer product hilbert space frames: Quantitative bounds, topological properties, and full geometric characterization. Journal of Mathematical Analysis and Applications, 441(1):475-498, 2016.

[11] P.G. Casazza and G. Kutyniok. Finite Frames: Theory and Applications. Applied and Numerical Harmonic Analysis. Birkhäuser Boston, 2012.

[12] Ole Christensen. An Introduction to Frames and Riesz Bases. Applied and Numerical Harmonic Analysis. Birkhäuser Boston, Inc., Boston, MA, 2003.

[13] Deguang Han, Keri Kornelson, David Larson, and Eric Weber. Frames for Undergraduates, volume 40 of Student Mathematical Library. American Mathematical Society, Providence, RI, 2007.

[14] Roger A. Horn and Charles R. Johnson, editors. Matrix Analysis. Cambridge University Press, New York, NY, USA, 1986.

[15] Roger A. Horn and Charles R. Johnson. Topics in Matrix Analysis. Cambridge University Press, Cambridge, 1991. 
[16] Gitta Kutyniok, Kasso A. Okoudjou, and Friedrich Philipp. Scalable frames and convex geometry. CoRR, abs/1310.8107, 2013.

[17] Albert W Marshall, Ingram Olkin, and Barry C Arnold. Inequalities: Theory of Majorization and its Applications, volume 143. Springer, 1979.

[18] Mátyás A Sustik, Joel A Tropp, Inderjit S Dhillon, and Robert W Heath. On the existence of equiangular tight frames. Linear Algebra and its applications, 426(2-3):619-635, 2007.

[19] Lloyd Welch. Lower bounds on the maximum cross correlation of signals (corresp.). IEEE Transactions on Information Theory, 20(3):397-399, 1974. 
Eric Pinkham was born May 25, 1987 in San Anselmo, California. He studied at The University of California, Santa Cruz from 2005 to 2009 where he earned a B.A. in mathematics. In 2012 he earned his M.A. in mathematics from San Francisco State University, graduating with honors. He attended the University of Missouri, Columbia from 2012 to 2017 where he was awarded the degree of Ph.D. in mathematics. 